\title{
Visual object tracking based on Motion- Adaptive Particle Filter under complex dynamics
}

\author{
Songxiao Cao* ${ }^{*}$, Xuanyin Wang and Ke Xiang
}

\begin{abstract}
This paper presents a novel particle filter called Motion-Adaptive Particle Filter (MAPF) to track fast-moving objects that have complex dynamic movements. The objective was to achieve effectiveness and robustness against abrupt motions and affine transformations. To that end, MAPF first predicted both velocity and acceleration according to prior data of the tracked objects, and then used a novel approach called subparticle drift (SPD) to improve the dynamic model when the target made a dramatic move from one frame to the next. Finally, the propagation distances of each direction in the dynamic model were determined based on the results of motion estimation and SPD. Experimental results showed that the proposed method was robust for tracking objects with complex dynamic movements and in terms of affine transformation and occlusion. Compared to Continuously Adaptive Mean-Shift (CAM-Shift), standard particle filter (PF), Velocity-Adaptive Particle Filter (VAPF), and Memory-based Particle Filter (M-PF), the proposed tracker was superior for objects moving with large random velocities and accelerations.
\end{abstract}

Keywords: Motion-adaptive model, Object tracking, Particle filter, Sub-particle drift

\section{Introduction}

Visual object tracking is becoming more and more important in many application fields nowadays, such as surveillance, robots, and human-computer interfaces [1-3]. However, it is still a challenging task to achieve reliable tracking due to erratic motions, occlusions, crowded background scenes, and different illuminations.

Many algorithms and methods have been proposed to track moving objects in video sequence, among which the mean shift (MS) and particle filter (PF) are frequently used. As a powerful tool in dealing with the non-linear and non-Gaussian problems, PF has been extensively studied for visual object tracking [4-9].

\subsection{Motion-adaptive problem of tracking}

Most PF-based trackers use the linear Gaussian dynamic model as their target motion model. However, this simple model cannot match the complexity of a fast-moving object with random velocity and acceleration.

\footnotetext{
* Correspondence: caosongxiao@zju.edu.cn

State Key Laboratory of Fluid Power and Mechatronic Systems, Zhejiang University, Hangzhou, China
}

This research aims to develop a robust tracker that could stably track a fast-moving rigid object with an unknown complexity dynamic model, which means that, even the target moves rapidly and changes abruptly in the moving direction, velocity, and acceleration, the tracker will still keep up with the target closely. To that end, the main focus was put on propagating the particles in a better way so as to make them fit the non-linear, non-Markov, and timevariant dynamic, because an accurate propagating region is important not only for the accurate estimation of the target state, but also for the recovery from tracking loss caused by occlusions. This paper proposed a novel PF called Motion-Adaptive Particle Filter (MAPF), which could predict both velocity and acceleration based on the past data of the tracked object, and introduced a novel method called subparticle drift (SPD) to improve the performance of the tracker during the target' dramatic motion from one frame to the following. The SPD was done by weighting samples of a main particle set and four sub-particle sets (left, right, up, and down sides of 
the main particle set), as well as the drift to the direction with a higher weight. A MAPF moving-object tracking was implemented in the adaptive color-based particle filter [6], which can also be used in many other particle filters where the velocity and acceleration of tracked objects should be considered.

\subsection{Related works}

In order to successfully track visual objects with complex dynamic movements, several extensions of the PF have been proposed including the random walk model and some other adaptive models. These PF-based trackers can be categorized as follows.

First, a training and learning process was employed for generating a dynamic model. Isard and Blake [10] used learned dynamics in the CONDENSATION algorithm to track moving objects. North and Blake [11] also got a dynamic model through learning to use the Expectation-Maximization CONDENSATION (EM-C) algorithm. Besides, Hess and Fern [12] proposed a discriminating training method for PFs. All of these methods may have good performance in tracking objects that have similar dynamic models with the trained video sequences. However, they are not very effective in tracking the arbitrarily moving objects which have not been previously learned, because the parameters used in the dynamic model are set to be constant values according to the trained video sequences. As a result, if the tracking object in a new test video sequence moves much faster or slower than the trained dynamic model, the tracking will end up with failure.

Second, a fixed constant-velocity model with fixed noise variance was used in a number of target trackers $[13,14]$ to propagate the particles between the frames. The result showed that a small velocity factor would lead to tracking loss when the target moved quickly, while, in case of a slow motion, it would cause drifting to background which was similar to the target.

Third, some adaptive strategies have been proposed to deal with the complex dynamics. Zhou [15] used an adaptive-velocity model, where the adaptive motion velocity was predicted using a first-order linear approximation based on the appearance difference between the incoming observation and the previous particle configuration, and the equation $\theta \mathrm{t}=\theta \mathrm{t}-1+\nu \mathrm{t}$ $+U \mathrm{t}$ was used as the adaptive state transition model, where $\nu \mathrm{t}$ is the predicted shift in the motion vector and $U \mathrm{t}$ is the adaptive noise parameter. Lui [16] determined the amount of observation noise based on the temporal difference of each state parameter and formulated the noise propagation distance in the dynamic model as $\Sigma_{u}=\max (a+b(u t-u t-1))$, where $a$ ensures that a minimum amount of noise will be added to an observation, and $b$ weighs the temporal difference, which is set to be a constant value. Del Bimbo [17] proposed a PF-based tracker to exploit a first order dynamic model and continuously adapt the model noise so as to balance uncertainties between the static and dynamic components of the state vector, which was formulated as $\theta t=A \theta t-1+\nu \mathrm{t}-1$, where $v \mathrm{t}-1$ is an additive, zero mean, and isotropic Gaussian uncertainty term. All the three papers used the velocity-adaptive model as the system dynamic model, called Velocity-Adaptive Particle Filter (VAPF). A Memory-based Particle Filter (M-PF) proposed by Mikami [18] introduced a paradigm called memorybased prior prediction which used the prior data of a target to predict prior distribution. The main idea of $\mathrm{M}-\mathrm{PF}$ is to predict the prior distribution of the target state in future steps by weighing samples of the past sequence of target states, where the sample weight is determined based on the long-term dynamics of the targeted system. These approaches can effectively track targets that move relatively quickly, since they automatically change the propagation distance according to the estimated velocity. All of the algorithms can only adapt to small velocity changes of the target, but, when the target moves with a large acceleration, changing position, pose, and size dramatically from one frame to the following, they tend to end up with failure.

Fourth, spatial and temporal information or multiscale strategies have been used in some other trackers. Yang [19] proposed a new search method for the estimation of consistent motion and disparity based on particle filtering, where the state dynamics involves the states of the neighboring blocks located in both spatial and temporal neighborhoods, which is similar to the method in this paper. However, Yang's method focuses on estimation of consistent motion and is used in applications such as image reconstruction and video coding, making it less suitable for tracking targets with large velocity and acceleration and unknown complex dynamics, which is the target of the present research. Han [20] used an analytic approach to better approximate and propagate density functions with a mixture of Gaussians. This approach used unscented transformation to derive a mixture representation of the predicted probable distribution function and applied a multistage sampling strategy to approximate the likelihood function. However, the random walk model was still used as the process model, which can only deal with simple and slow motions of objects, but is not appropriate for complex dynamics.

In contrast to the existing approaches mentioned above, the novel MAPF proposed in this research can predict both velocity and acceleration according to 
past data of the tracked object, and the introduced SPD can improve the performance when the target moves dramatically from one frame to the following. Experimental results showed that the proposed method was robust for tracking objects with complex dynamic movements and in terms of affine transformation and occlusion.

\subsection{Paper organization}

Section 2 summarizes related methods used for visual object tracking; Section 3 deals with the MAPF; Section 4 introduces experimental results and makes comparisons with the Continuously Adaptive Mean-shift (CAM-Shift) [21], standard PF [6], VAPF [15, 16], and M-PF [18]; and Section 5 outlines the conclusions and suggestions for future research.

\section{Color-based PF}

This section briefly overviews the main concepts of related methods that are discussed in this paper, including the color distribution model used in all of the discussed methods, the basic formulae of the PF used for visual tracking, and the velocity-adaptive model.

\subsection{Color distribution model}

To achieve robustness against non-rigidity, rotation, and partial occlusion, the color distribution is a widely used target representation model. Because the intensity channel $\mathrm{V}$ is susceptible to illumination variations, a lot of models choose histograms of $h \times s$ bins in H-S two-dimension color space to represent the color distribution. To increase the reliability of the color distribution when boundary pixels resembles the background of the occluded, smaller weights are assigned to the pixels that are further away from the region center by employing a weighting function:

$$
k(r)=\left\{\begin{array}{lll}
1-r^{2} & : & r<1 \\
0 & : & \text { otherwise }
\end{array}\right.
$$

The color distribution $p_{y}=\left\{p_{y}^{(u)}\right\} u=1 \ldots m$ at location $y$ is calculated as:

$$
p_{y}^{(u)}=C h \sum_{i=1}^{n_{h}} k\left(\left\|\frac{y-x_{i}}{a}\right\|\right) \delta\left[b\left(x_{i}\right)-u\right]
$$

where $\left\{x_{i}\right\}_{i=1 \ldots n h}$ is the normalized pixel location of the target candidate, $m=16 \times 8$ is the bin used in the color distribution in this paper, $n_{h}$ is the number of pixels in the region, $b\left(x_{i}\right)$ is the function which assigns the color at location $x_{i}$ to the corresponding bin, $a$ is the

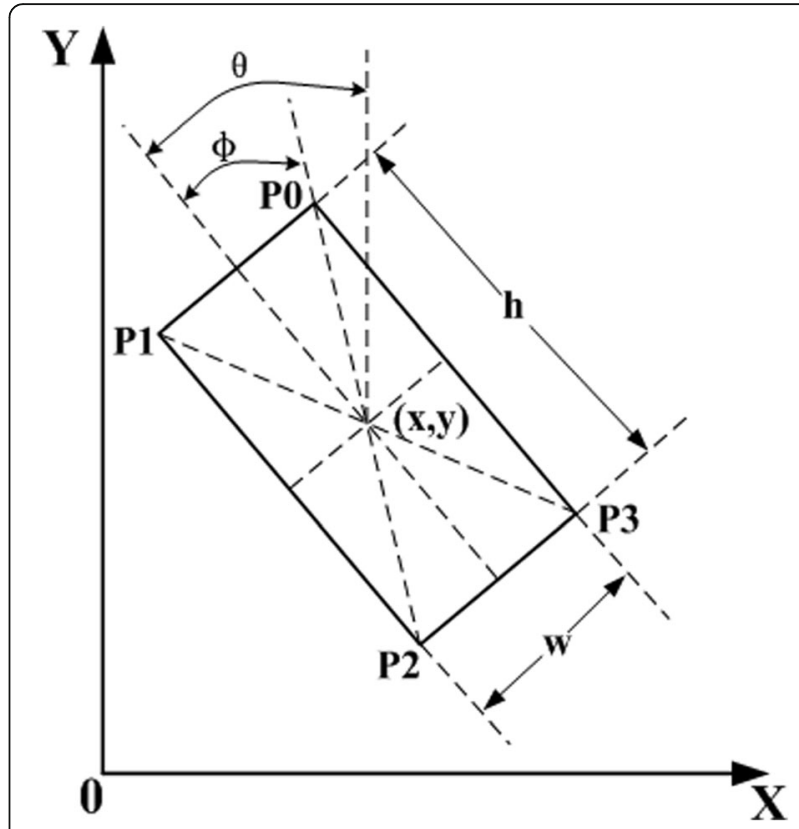

Fig. 1 Rectangular template model

parameter used to adapt the size of the region, and $\delta$ is the Kronecher delta function. The normalization constant is calculated as:

$$
C h=\frac{1}{\sum_{i=1}^{n_{h}} k\left(\left\|\frac{y-x_{i}}{a}\right\|\right)}
$$

which ensures that $p(y)=\sum_{u=1}^{m} p_{u}(y)=1$. Because the $p_{u}(y)$ is the normalized distribution, its total sum should be 1 .

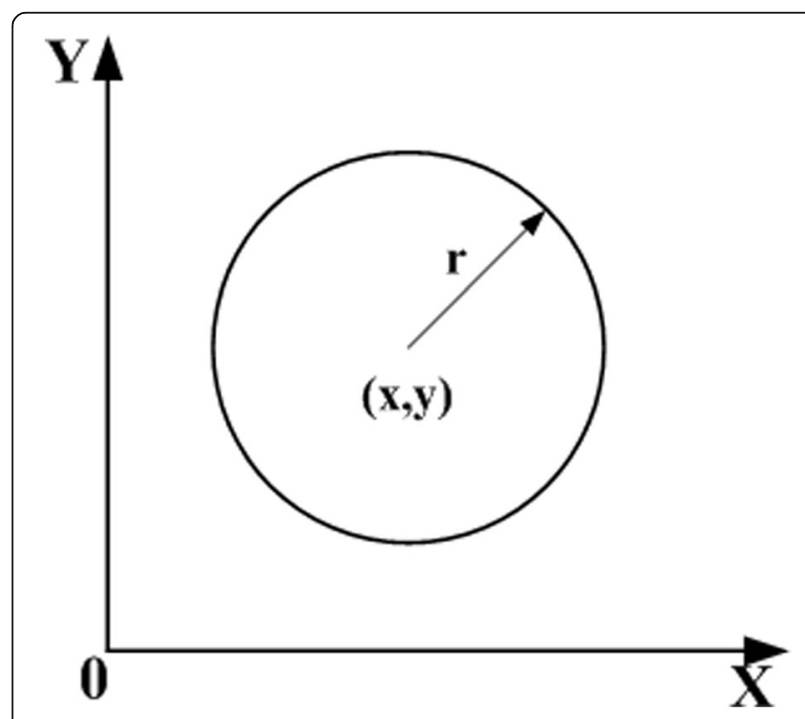

Fig. 2 Circular template model 


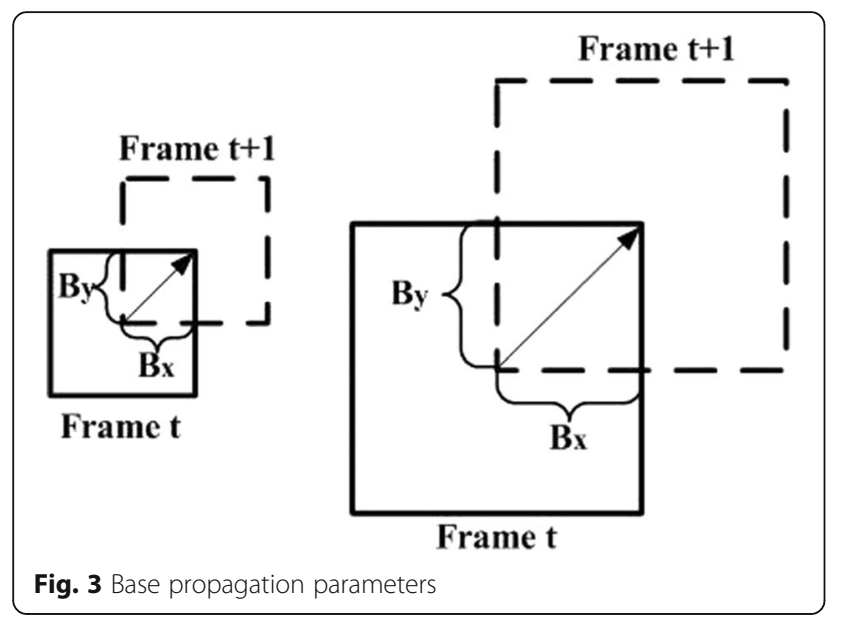

To measure the similarity between distributions $p$ $=\left\{p_{u}\right\} u=1 \ldots m$ and $q=\left\{q_{u}\right\} u=1 \ldots m$, where $m$ is the number of bins, the Bhattacharyya distance is adopted:

$$
d=\sqrt{1-\rho[p, q]}
$$

where

$$
\rho[p, q]=\sum_{u=1}^{m} \sqrt{p_{u} q_{u}}
$$

\subsection{Particle filter}

$\mathrm{PF}$ is a Monte Carlo approximation to the optimal Bayesian filter and provides robust tracking of moving objects in a cluttered environment, especially in the case of non-linear and non-Gaussian problems where the interest lies in the detection and tracking of moving objects. It is a probabilistic framework for sequentially estimating the target's state to recursively computer the posterior density $p\left(s_{t} \mid z_{1: t}\right)$ of current object state $s_{t^{-}}$ conditioned on all observations $z_{1: t}=\left(z_{1}, z_{2} \ldots \ldots . z_{t}\right)$ up to time $t$.

To implement a standard PF, a state representation $s_{t}$ should be identified that, in object tracking, might include object locations, scales, and rotations. Moreover, it is necessary to design three distributions: the process $d y-$ namical distribution $p\left(s_{t} \mid s_{t-1}\right)$, which describes how the object moves between frames; the proposal distribution $q\left(s_{t} \mid s_{1: t-1}, z_{1: t}\right)$, which is sampled each time the particle distribution updates; and the observation likelihood distribution $p\left(z_{t} \mid s_{t}\right)$, which means how the object appears in the video frame.

This paper focuses on the dynamical distribution $p\left(s_{t} \mid\right.$ $\left.s_{t-1}\right)$, which can usually be represented as a linear stochastic differential function:

$$
s_{t}=A s_{t-1}+B \omega_{t-1}
$$

where $A$ defines the deterministic component of the dynamic model; $s_{t}$ is the state vector of time $t ; \omega_{t-1} \in(0,1)$ is the system noise, which is usually a uniformly random variable or a multivariate Gaussian random variable; and $B$ is the propagation distance, indicating how far away the particles can propagate in the next frame and determining the tracking performance when the object makes an arbitrary move.

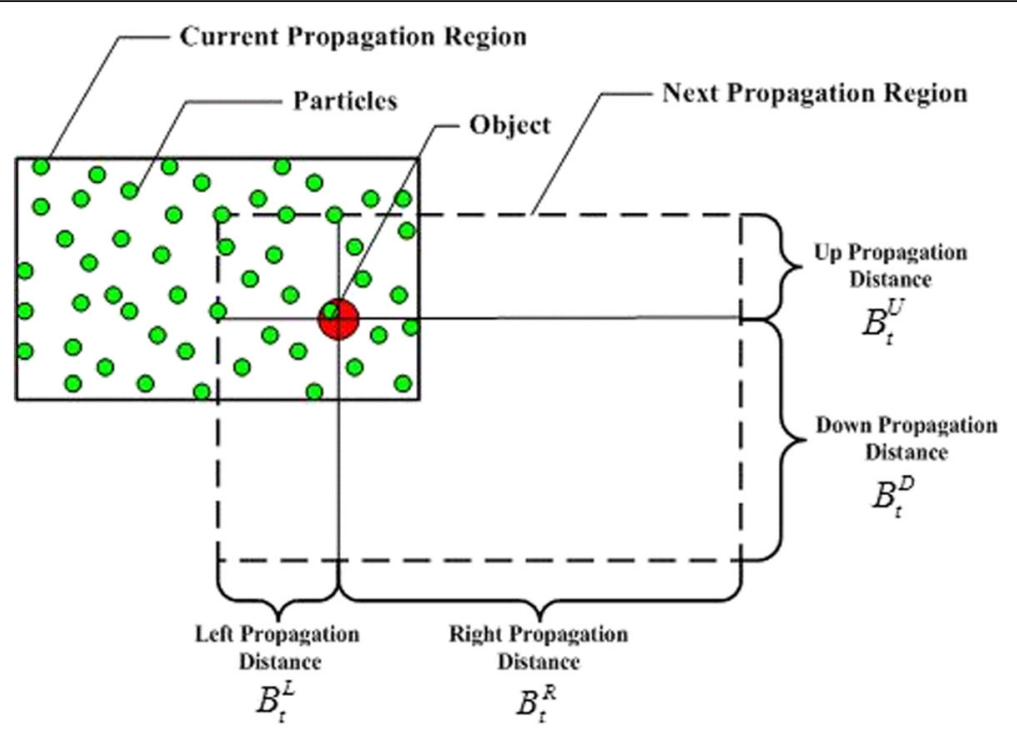

Fig. 4 Particle propagation model 

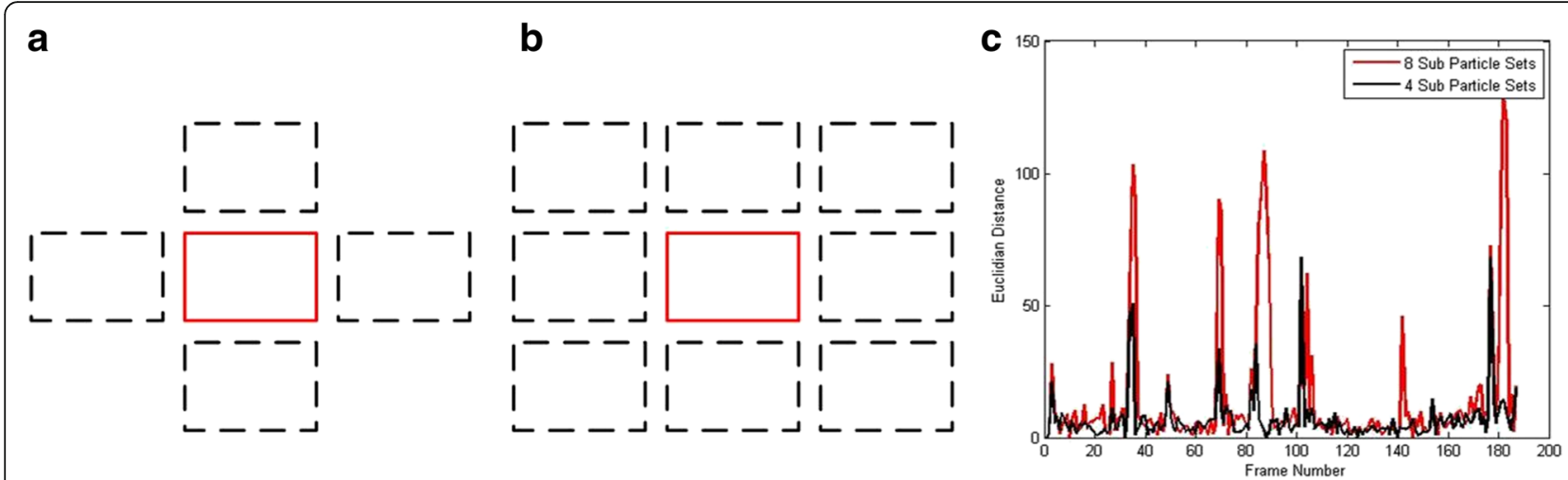

Fig. 5 a Four sub particle sets, b eight particle sets, and c Euclidian distance of "basketball_2" using eight sub-particle sets and four sub-particle sets

As mentioned before, there are three different methods in dealing with the dynamic model: training and learning, a fixed constant-velocity model, and a velocity adaptive model. In the training and learning method, the propagation distance $B$ is determined through training and learning using some video sequences, while, in a fixed constant-velocity model, $B$ is set to be a constant value during the tracking process, and, as an adaptive method, the velocity adaptive model (VAPF) updates the propagation distance according to the temporal difference of previous frames by calculating the average velocity:

$$
\begin{aligned}
& \overline{s_{t}}=\frac{1}{j} \sum_{n=t-j}^{t}\left|s_{n}-s_{n-1}\right| \\
& B_{t}^{\prime} \propto \overline{s_{t}^{\prime}}
\end{aligned}
$$

where $\overline{s_{t}^{\prime}}$ means the average state velocity in the previous $j$ frames of one certain dimension, and $B_{t}^{\prime}$ is the corresponding propagation distance in that dimension. For example, in the $x$ direction, $B_{t}^{x} \propto \overline{x_{t}}=\frac{1}{j} \sum_{n=t-j}^{t}\left|x_{n}-x_{n-1}\right|$.

The main advantage of the PFs mentioned above is its robust performance under clutter background or occlusion. The main drawback is that it still cannot effectively track objects moving with rapid speeds and large accelerations.

\section{The proposed method}

This section describes the proposed MAPF used for visual object tracking in complex dynamics, including the template model of the tracked objects (bounding rectangular model and circular model), and the proposed motion-adaptive model.

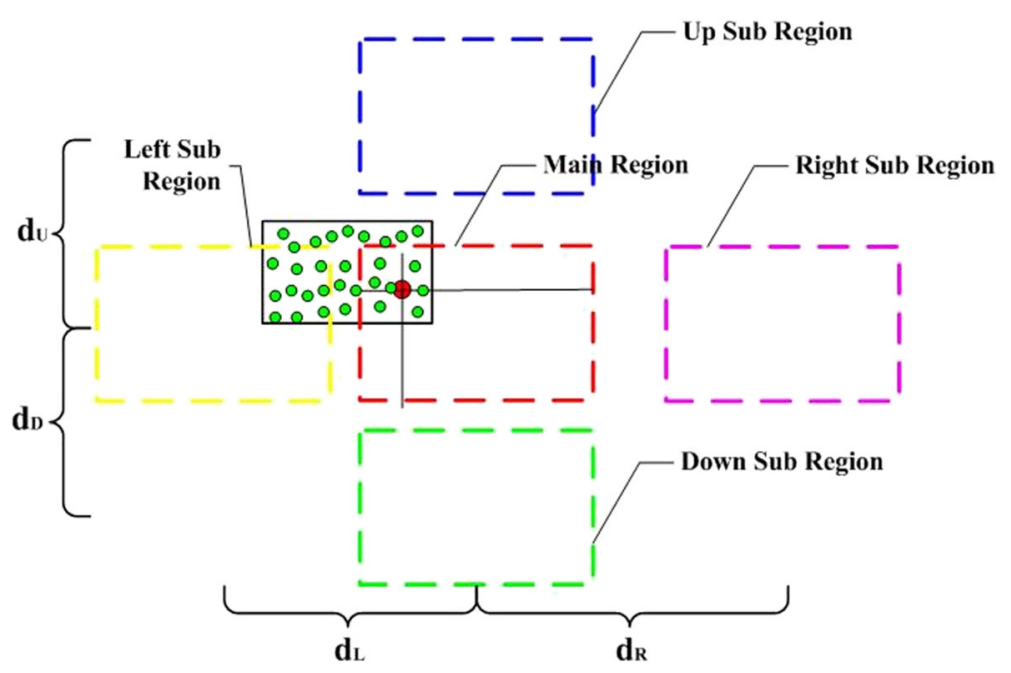

Fig. 6 Sub-particle model 


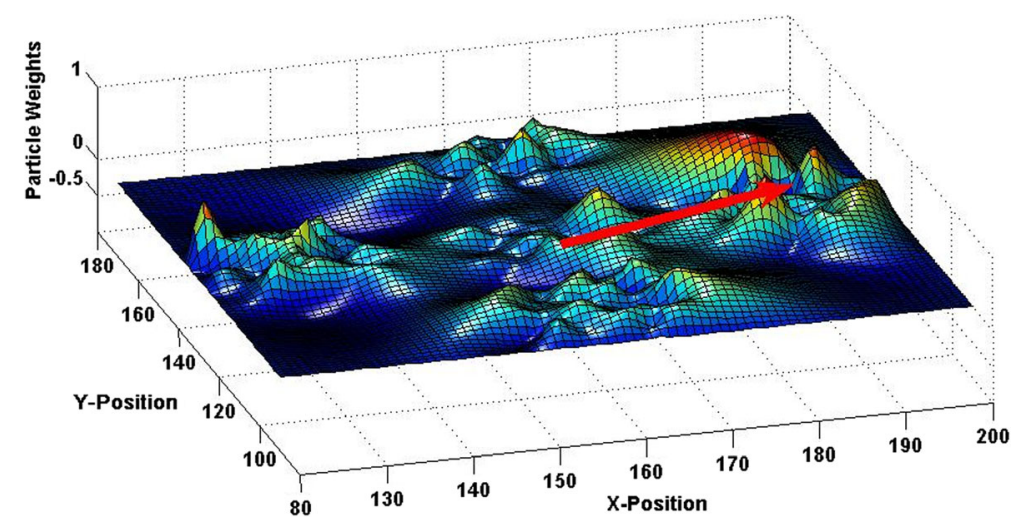

Fig. 7 Sub-particle drift model

\subsection{Template model}

As the proposed method is based on adaptive color-based $\mathrm{PF}$, the tracking process is accomplished by choosing an appropriate bounding region as the area of interest out of some candidate regions and comparing the similarity of the color distribution between each other. In this paper, two different template modes, the rectangular model and circular model, were used according to the tracked object's template.

\subsubsection{Rectangular template model}

In this model, a candidate bounding box can be described as:

$$
s=[x, y, w, h, \theta]^{\mathrm{T}}
$$

where $x, y$ represents the location of the rectangle, $w, h$ the width and height of the rectangle, and $\theta$ the rotation angle, as is described in Fig. 1. Thus, the track region
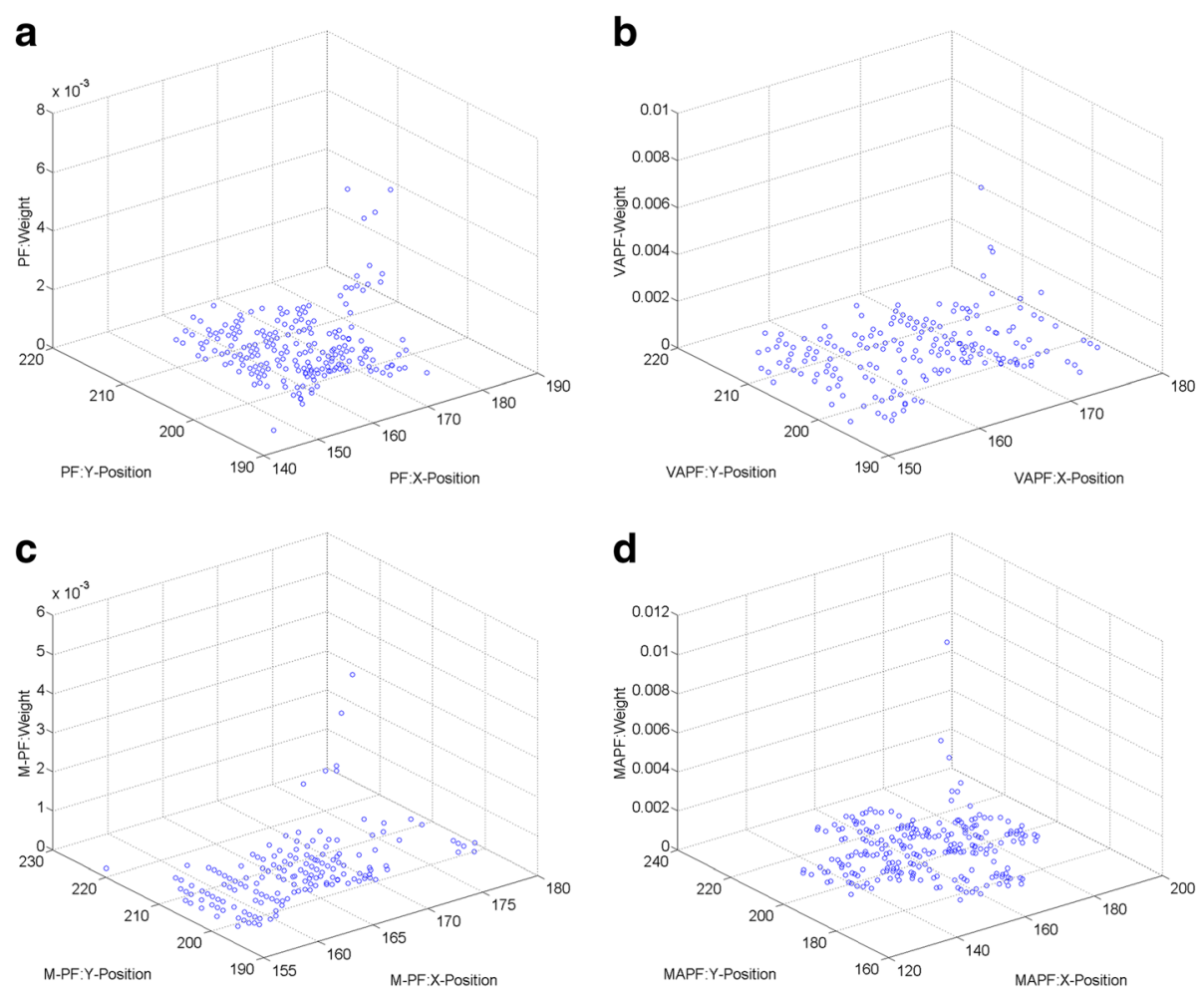

Fig. 8 Particle weights distribution of the 7th frame of video "tennis" using different algorithms, PF(a), VAPF(b), M-PF(c) and Proposed(d) 

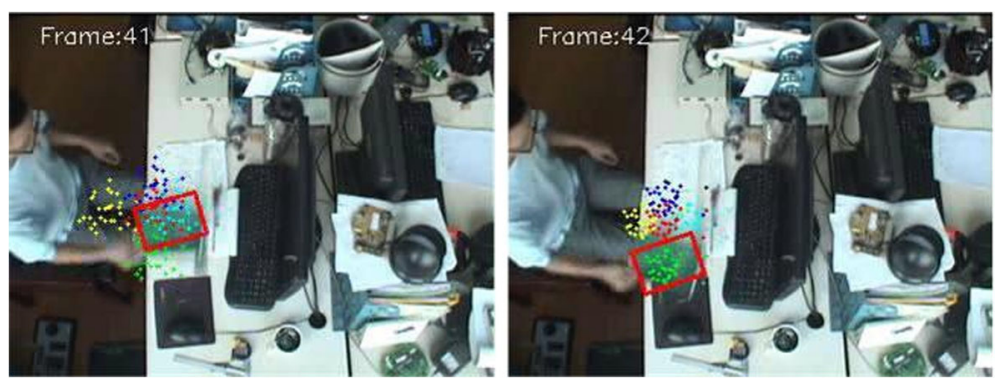

Fig. 9 Sub-particle drift example

can be represented by four vertices $\left\{P_{0}, P_{1}, P_{2}, P_{3}\right\}$ of the rectangle, of which the corresponding coordinates $\operatorname{are}\left\{\left(x_{0}, y_{0}\right),\left(x_{1}, y_{1}\right),\left(x_{2}, y_{2}\right),\left(x_{3}, y_{3}\right)\right\}$ :

$$
\begin{aligned}
& {\left[\begin{array}{l}
x_{0} \\
x_{1} \\
x_{2} \\
x_{3}
\end{array}\right]=\left[\begin{array}{l}
x \\
x \\
x \\
x
\end{array}\right]+\left[\begin{array}{l}
R \sin (\phi-\theta) \\
R \sin (\pi-\phi-\theta) \\
R \sin (\pi+\phi-\theta) \\
R \sin (-\phi-\theta)
\end{array}\right]} \\
& {\left[\begin{array}{l}
y_{0} \\
y_{1} \\
y_{2} \\
y_{3}
\end{array}\right]=\left[\begin{array}{l}
y \\
y \\
y \\
y
\end{array}\right]+\left[\begin{array}{l}
R \cos (\phi-\theta) \\
R \cos (\pi-\phi-\theta) \\
R \cos (\pi+\phi-\theta) \\
R \cos (-\phi-\theta)
\end{array}\right]}
\end{aligned}
$$

where $R=\frac{1}{2} \sqrt{w^{2}+h^{2}}, \phi=\arctan (w / h)$, and $-\frac{\pi}{2} \leq \theta \leq \frac{\pi}{2}$.

The state variable $s$ can be separated into two parts, $s^{p}$ $=[x, y]^{\mathrm{T}}$ and $s^{a}=[w, h, \theta]^{\mathrm{T}}$, where $s^{p}$ represents the object's position-related parameters, while $s^{a}$ represents the affine transformations.

\subsubsection{Circular template model}

The circular model is relatively simple, in which a candidate bounding circle can be described as:

$$
s=[x, y, r]^{\mathrm{T}}
$$

where $x, y$ represents the location of the circle and $r$ the radius of the circle, as is described in Fig. 2. Then the tracking region can be represented by a circle centered in $(x, y)$ with the radius $r$. As has been described above, the state variable $s$ is separated into two parts, $s^{p}=[x, y]^{\mathrm{T}}$ and $s^{a}=[r]^{\mathrm{T}}$, where $s^{p}$ represents the object's position-related parameters, while $s^{a}$ represents the affine transformations.

\subsection{Motion-adaptive model}

In order to follow the tracked object moving with a complex dynamic, a motion adaptive model was proposed that combined velocity and acceleration estimation with a new approach called sub-particle drift.

\subsubsection{Velocity and acceleration estimation}

Setting $v_{t}^{p}$ as the velocity vector at time $t$ of the positionrelated parameter and $a_{t}^{p}$ the acceleration vector, the following formulae can be obtained:

$$
\begin{aligned}
& v_{t}^{p}=d s^{p} / d t=\left(s_{t}^{p}-s_{t-1}^{p}\right) / T \\
& a_{t}^{p}=d v^{p} / d t=\left(s_{t}^{p}-2 s_{t-1}^{p}+s_{t-2}^{p}\right) / T^{2}
\end{aligned}
$$

where $T$ is the sampling time and $\lambda T=1 / T$ :

$$
v_{t}^{p}=\lambda_{T}\left(s_{t}^{p}-s_{t-1}^{p}\right)
$$
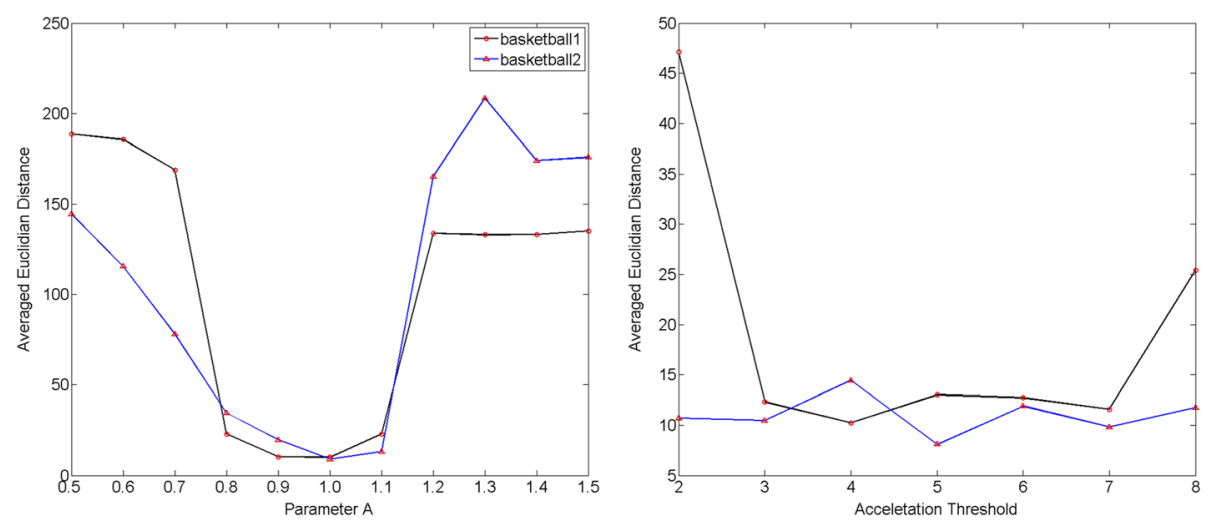

Fig. 10 The tracking results using different parameters 
Table 1 Resulting of using different particle number

\begin{tabular}{|c|c|c|c|c|c|}
\hline Sequences & Number of Particles & CAM-Shift & PF & VAPF & Proposed \\
\hline \multirow[t]{5}{*}{ Book } & 100 & 138.83 & 34.69 & 11.40 & 7.05 \\
\hline & 150 & & 34.99 & 18.68 & 9.99 \\
\hline & 200 & & 62.47 & 17.30 & 5.02 \\
\hline & 250 & & 35.15 & 77.15 & 5.41 \\
\hline & 300 & & 34.06 & 20.79 & 5.13 \\
\hline \multirow[t]{5}{*}{ Bottle } & 100 & 27.78 & 26.03 & 24.86 & 20.74 \\
\hline & 150 & & 25.83 & 22.90 & 20.96 \\
\hline & 200 & & 25.70 & 22.40 & 20.47 \\
\hline & 250 & & 26.18 & 22.17 & 20.15 \\
\hline & 300 & & 25.66 & 22.55 & 20.53 \\
\hline \multirow[t]{5}{*}{ Basketball_1 } & 100 & 78.58 & 71.09 & 53.59 & 14.90 \\
\hline & 150 & & 88.28 & 85.50 & 16.51 \\
\hline & 200 & & 88.76 & 44.12 & 9.93 \\
\hline & 250 & & 87.36 & 36.48 & 8.34 \\
\hline & 300 & & 65.65 & 65.83 & 8.35 \\
\hline \multirow[t]{5}{*}{ Basketball_2 } & 100 & 75.04 & 52.78 & 46.19 & 40.12 \\
\hline & 150 & & 47.58 & 35.83 & 16.29 \\
\hline & 200 & & 44.58 & 42.80 & 11.35 \\
\hline & 250 & & 42.41 & 33.40 & 11.55 \\
\hline & 300 & & 46.16 & 42.13 & 9.79 \\
\hline \multirow[t]{5}{*}{ Tennis } & 100 & 116.31 & 136.04 & 80.47 & 2.31 \\
\hline & 150 & & 15.24 & 13.96 & 2.27 \\
\hline & 200 & & 80.78 & 79.07 & 2.04 \\
\hline & 250 & & 80.47 & 79.24 & 2.18 \\
\hline & 300 & & 79.87 & 10.63 & 2.06 \\
\hline
\end{tabular}

$$
a_{t}^{p}=\lambda_{T}^{2}\left(s_{t}^{p}-2 s_{t-1}^{p}+s_{t-2}^{p}\right)
$$

To reduce random noises, a weighted function was used for the velocity $v_{t}^{p}$ and acceleration $a_{t}^{p}$ :

$$
\begin{aligned}
& \overline{v_{t}^{p}}=\lambda_{T} \sum_{n=0}^{N s} \alpha_{n} v_{t-n}^{p} \\
& \overline{a_{t}^{p}}=\lambda_{T}^{2} \sum_{n=0}^{N s} \beta_{n} a_{t-n}^{p}
\end{aligned}
$$

where $\alpha_{n}$ and $\beta_{n}$ are the normalization factors for every velocity and acceleration. And it was assumed that the older velocities and accelerations are assigned smaller weights:

$$
\left\{\begin{array} { l } 
{ \sum _ { n = 0 } ^ { N s } \alpha _ { n } = 1 } \\
{ \alpha _ { 0 } > \alpha _ { 1 } > \ldots . . > \alpha _ { n } }
\end{array} \quad \left\{\begin{array}{l}
\sum_{n=0}^{N s} \beta_{n}=1 \\
\beta_{0}>\beta_{1}>\ldots \ldots>\beta_{n}
\end{array}\right.\right.
$$

where $N s$ is the number of frames that needs to be smoothed.
Table 2 Parameter values used in the test

\begin{tabular}{llllll}
\hline Video & A & BBase & Ns & Ta & $\sigma$ \\
\hline Book & 1 & $(15,15,0.1,0.1,0.1)$ & 3 & 4 & 0.16 \\
Bottle & 1 & $(10,10,0.1,0.1,0.1)$ & 3 & 4 & 0.16 \\
Motinas_chamber & 1 & $(20,20,0.1,0.1,0.1)$ & 3 & 4 & 0.16 \\
Bolt & 1 & $(10,10,0.1,0.1,0.1)$ & 3 & 4 & 0.16 \\
Diving & 1 & $(10,10,0.1,0.1,0.1)$ & 3 & 4 & 0.16 \\
MountainBike & 1 & $(10,10,0.1,0.1,0.1)$ & 3 & 4 & 0.16 \\
RedTeam & 1 & $(10,10,0.1,0.1,0.1)$ & 3 & 4 & 0.16 \\
Basketball_1 & 1 & $(15,15,0.1)$ & 3 & 4 & 0.16 \\
Basketball_2 & 1 & $(15,15,0.1)$ & 3 & 4 & 0.16 \\
Tennis & 1 & $(5,5,0.1)$ & 3 & 4 & 0.16 \\
v_juggle_11_06 & 1 & $(10,10,0.1)$ & 3 & 4 & 0.16
\end{tabular}

Setting $B_{\mathrm{Base}}^{p}$ and $B_{\mathrm{Max}}^{p}$ as the base propagation and maximum propagation distance parameters of $s^{p}$, respectively, the following formulae can be obtained:

$$
\begin{cases}B_{\mathrm{Max}}^{p}=B_{\mathrm{Base}}^{p}\left(\frac{\overline{a_{t}^{p}}}{2}+1\right) & \text { if } \quad\left|\overline{a_{t}^{p}}\right|>T a \\ B_{\mathrm{Max}}^{p}=B_{\mathrm{Base}}^{p}\left(\frac{\overline{v_{t}^{p}}}{4}+1\right) & \text { else }\end{cases}
$$

Specifically, the base propagation distance $B_{\text {Base }}^{p}$, which consists of $\left(B_{x}, B_{y}\right)$, is decided automatically according to the size of the tracked object. Take the rectangular template model as an example, as is seen in Fig. 3.

$\mathrm{Ta}$ is the acceleration threshold, which means that, when the acceleration at time $t$ is larger than the threshold, the propagation radius is determined by $\overline{a_{t}^{p}}$, while when the acceleration is smaller, it is determined by $\overline{v_{t}^{p}}$.

Table 3 Video sequences with different attributes

\begin{tabular}{lllll}
\hline Attribute video & OCC & BC & AT & AM \\
\hline Motians_chamber & $\checkmark$ & & & \\
Bottle & & $\checkmark$ & \\
Book & & $\checkmark$ & $\checkmark$ \\
Basketball_1 & & & $\checkmark$ \\
Basketball_2 & $\checkmark$ & & $\checkmark$ \\
Basketball_3 & $\checkmark$ & $\checkmark$ & $\checkmark$ \\
Tennis & & & $\checkmark$ \\
v_juggle_11_06 & $\checkmark$ & $\checkmark$ & & $\checkmark$ \\
v_juggle_15_02 & $\checkmark$ & $\checkmark$ & & $\checkmark$ \\
Bolt & & & $\checkmark$ \\
Diving & & & $\checkmark$ & \\
MountainBike & & $\checkmark$ & \\
RedTeam & & & \\
\hline
\end{tabular}




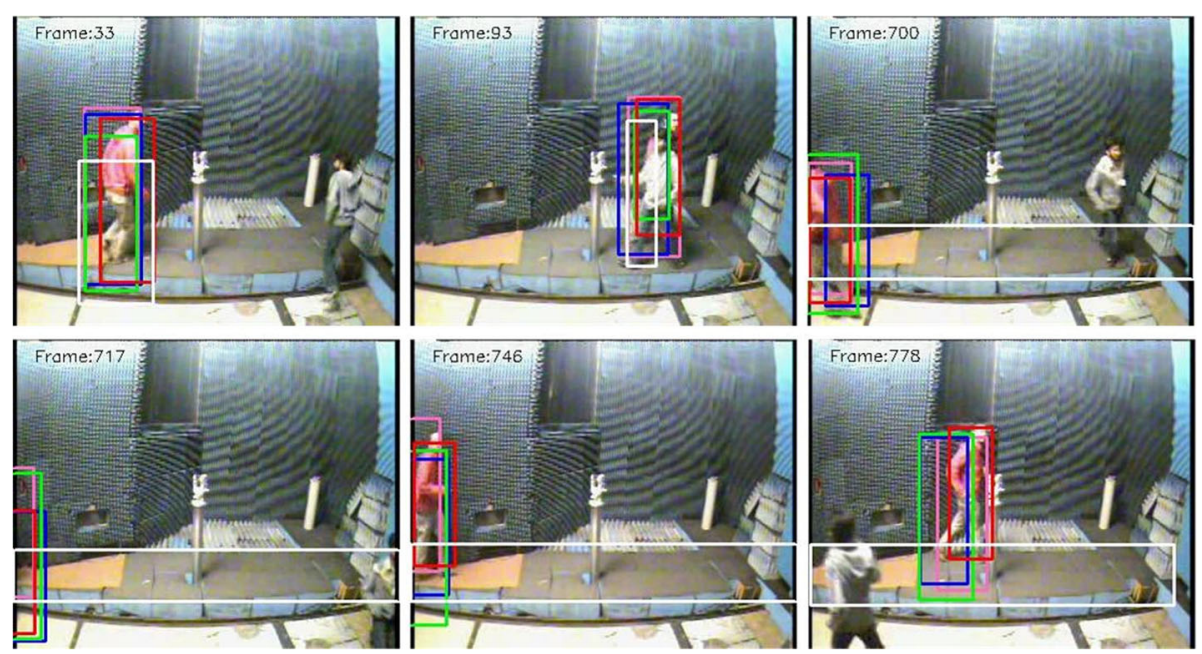

Fig. 11 The tracking results for the video "motinas_chamber" using CAM-Shift (white), PF (blue), VAPF(green), M-PF(pink), and the proposed tracker (red). Particle number $N=200$

The algorithm updates the propagation distance of each frame according to the $\overline{v_{t}^{p}}$ and $\overline{a_{t}^{p}}$,

$$
B_{t}^{p}=\left[\begin{array}{cc}
B_{t}^{x} & 0 \\
0 & B_{t}^{y}
\end{array}\right]=\left[\begin{array}{cc}
B_{t}^{L}+B_{t}^{R} & 0 \\
0 & B_{t}^{U}+B_{t}^{D}
\end{array}\right]
$$

while the affine transformation-related parameters $B_{t}^{a}$ are all set to be one constant value. $B_{t}^{L}, B_{t}^{R}, B_{t}^{U}$, and $B_{t}^{D}$ are the propagation distances of respectively the left, right, up, and down side based on the position of the object in the current frame, as is described in Fig. 4.

And the distance of each side is determined based on the velocity or acceleration estimation. For example, in the horizontal movement, when the velocity or acceleration is negative, that is, the object tends to move to the left side, the left propagation distance is set to be:

$$
B_{t}^{L}=\left\{\begin{array}{lll}
B_{\mathrm{Max}}^{x}=B_{x}\left(\frac{\overline{a_{t}^{x}}}{2}+1\right) & \text { if } & \left|\overline{a_{t}^{x}}\right|>T a \\
B_{\mathrm{Max}}^{x}=B_{x}\left(\frac{\overline{v_{t}^{x}}}{4}+1\right) & \text { else }
\end{array}\right.
$$

while, in the opposite direction, the right propagation distance $B_{t}^{R}=B_{\text {Base }}^{x}$.

Then, the dynamic model at time $t$ can be represented as:

$$
s_{t}=A s_{t-1}+B_{t} \omega_{t-1}
$$
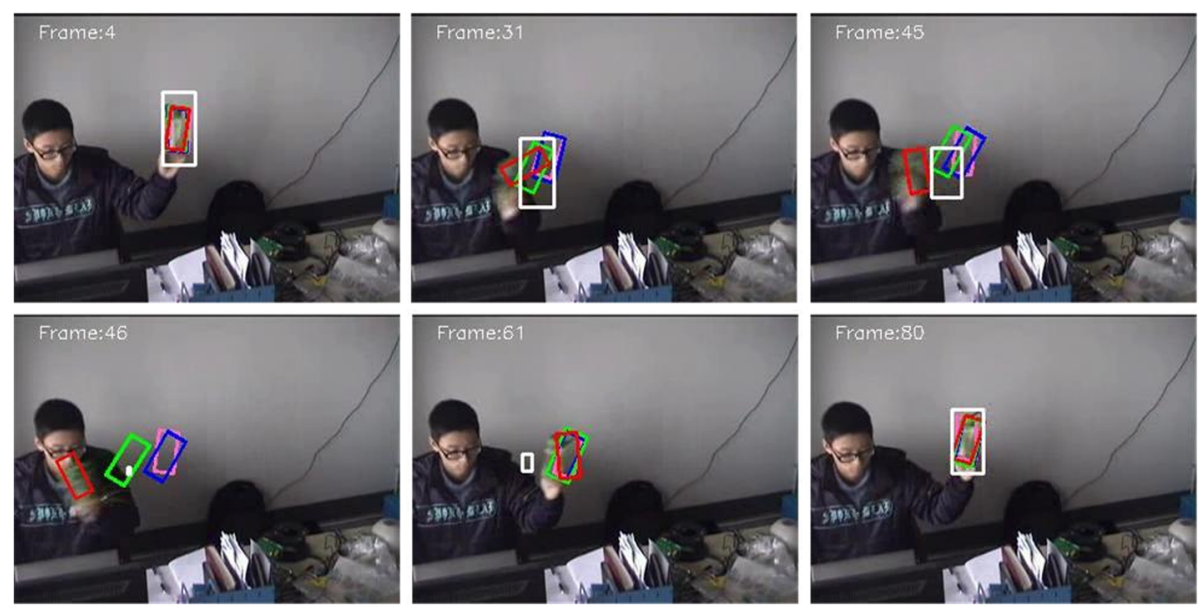

Fig. 12 The tracking results for the video "bottle" using CAM-Shift (white), PF (blue), VAPF (green), M-PF (pink), and the proposed tracker (red). Particle number $N=200$ 


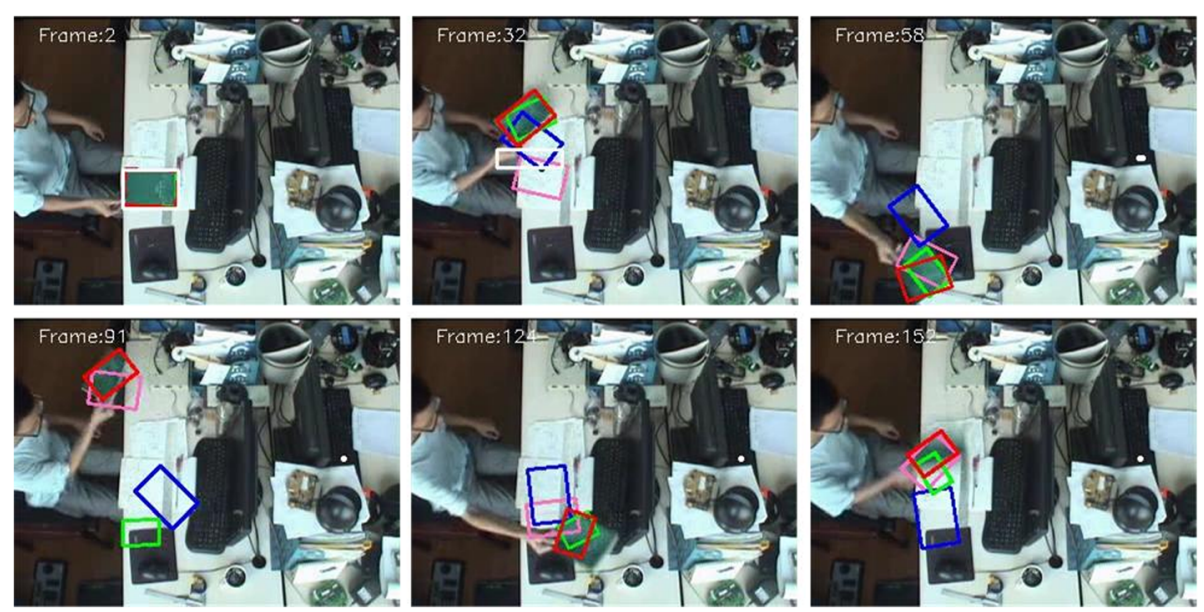

Fig. 13 The tracking results for the video "book" using CAM-Shift (white), PF (blue), VAPF (green), M-PF (pink), and the proposed tracker (red). Particle number $N=300$

\subsubsection{Sub-particle drift}

In order to enhance the robustness of this research's tracker for objects moving more dramatically, a sub-particle drift method is proposed. In this method, the center of the main particle set region drifts to the sub-particle set in which the total particle weight $\sum_{i=0}^{N} w_{i}$ is the largest.

The sub-particle drift process is achieved by comparing the particle weights and drifting to the maximum in the next frame, as is described in the following formula:

$$
w_{\max }=\max \left\{\sum_{i=0}^{N} w_{i}^{M}, \sum_{i=0}^{N} w_{i}^{S P 0} \ldots \ldots \sum_{i=0}^{N} w_{i}^{S P n}\right\}
$$

$$
\left[\begin{array}{l}
x_{t+1} \\
y_{t+1}
\end{array}\right]=\left[\begin{array}{l}
x_{t}^{\max } \\
y_{t}^{\max }
\end{array}\right]
$$

where $w_{i}^{M}$ and $w_{i}^{S P n}$ represent the particle weights of the main and $n^{\text {th }}$ sub particle region, respectively, and $x_{t}^{\max }, y_{t}^{\max }$ are the $x$ and $y$ position with the largest weight determined by Eq. (24). For example, when the second sub-particle set has the largest weight, $x_{t}^{\max }=x_{t}^{S P 1}$ and $y_{t}^{\max }=y_{t}^{S P 1}$. Therefore, when the maximum weight is in the main particle region, the propagation process is achieved according to Eq.(23); on the other hand, when the maximum region is one of the sub-particle sets, the dynamic model changes to the following:

$$
s_{t}=A s_{t-1}^{\max }+B_{t} \omega_{t-1}
$$
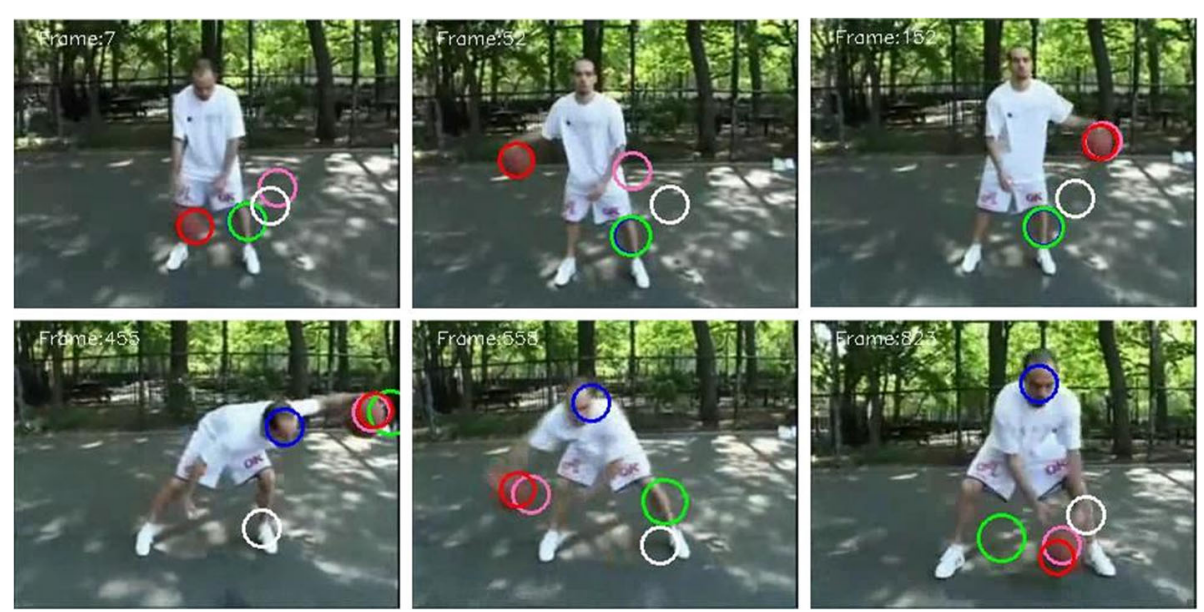

Fig. 14 The tracking results for the video "basketball_1" using CAM-Shift (white), PF (blue), VAPF (green), M-PF (pink), and the proposed tracker (red). Particle number $N=250$ 
where $s_{t-1}^{\max }$ is the one that has the maximum weight in Eq. (24).

MAPF algorithm

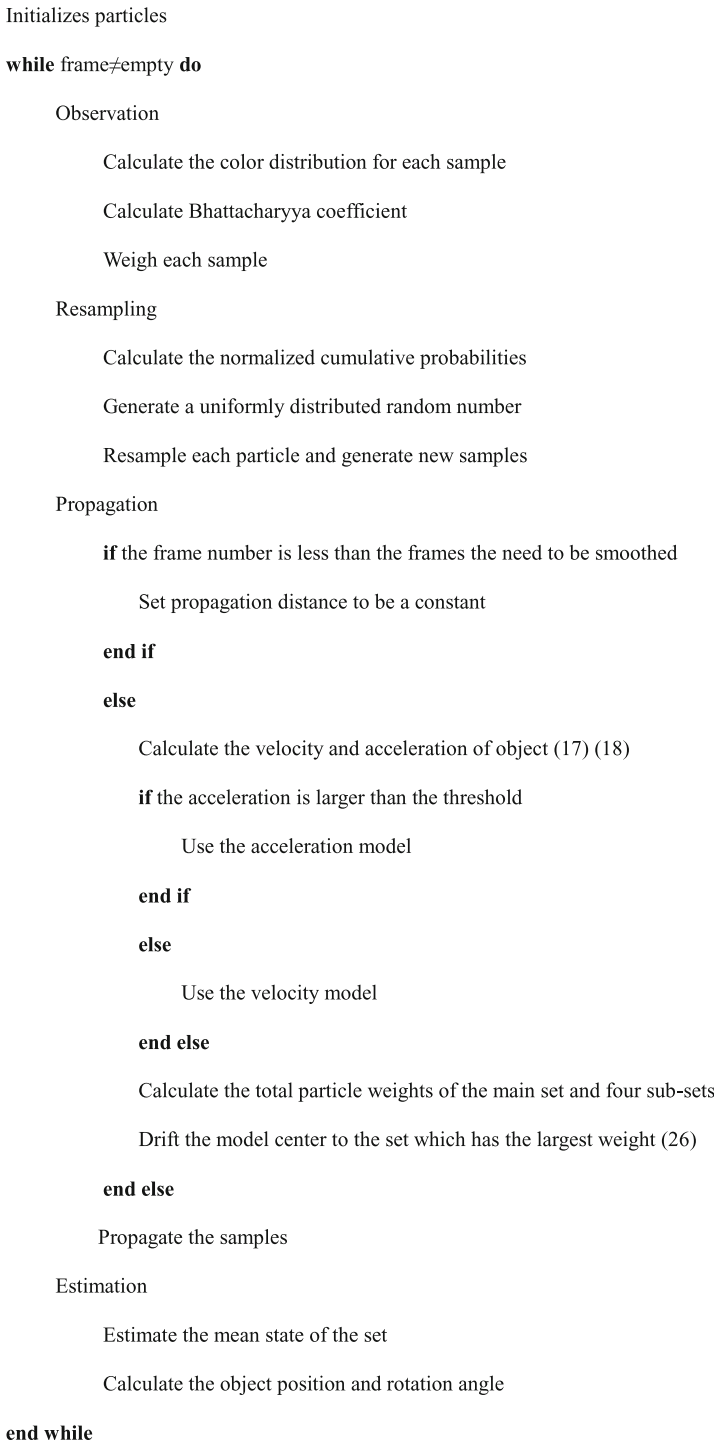

\section{Results and discussion}

To demonstrate the effectiveness and robustness of the proposed tracking scheme, 13 different color videos were used in the experiments (Additional files 1-13), which were obtained from different datasets. Four of them (Bolt, Diving, MountianBike, Redteam) belonged to the tracking benchmark dataset [22], three (basketall_1, basketall_2, basketall_3) were clips of the famous basketball dribbling teaching movie Bobbito's Basics to Boogie, two (v_juggle_11_06, v_juggle_15_02) from UCF
YouTube Action Dataset [23], one (motians_chamber) from Surveillance Performance Evaluation Initiative (SPEVI) dataset [24], and the rest (book, bottle, tennis) were acquired indoors using a SONY CCD camera EX-FCB48. These videos posed several challenges, such as objects moving at a high speed and large acceleration, affine transformation, partial or total occlusion, and images with colors similar to the objects in the background. For all the videos, the target object was manually selected in the first frame, and so was the corresponding template model.

The proposed tracking method was also compared with the four existing trackers, CAM-Shift, PF, APF, and $\mathrm{M}-\mathrm{PF}$, to identify their correlations. All the algorithms were implemented in $\mathrm{C}++$ using the OpenCV library and run on a $1.8 \mathrm{GHz}$ Pentium Dual-Core CPU, with 2Gbyte DDR memory. The parameter $T$ in Eq. (13) was $T=1 / 25$ seconds because the video format was PAL.

\subsection{Parameters selection}

There are some parameters that can affect the performance of the proposed tracker. In order to achieve the best tracking performance, the process of selecting some parameters is discussed as follows.

\section{Number of the sub-particle set}

Based on the concept of sub-particle drift and the fact that the object will be moving in horizontal and vertical directions, several strategies such as the four sub-particle sets and the eight sub-particle sets, as is seen in Fig. 5a, b, were considered and evaluated first to identify the best one. Figure $5 \mathrm{c}$ shows the evaluation result of using the four particle sets and the eight sub-particle sets. The evaluation metric of the Euclidian distance will be detailed in section 4.3.1. The figures indicate that the four sets strategy shows a slight advantage in tracking position accuracy. The reason is that, with the same particle number, more sub-particle sets will cause fewer particles in each set, making the distribution more sparse, which means the prior distribution is not as accurate as that of the four particle sets. Another reason is that, if the object moves only in the horizontal or vertical direction, the four surrounding sub-particle sets are enough to keep tracking it when high speed or large acceleration takes place. However, if the object moves diagonally, it will have the "resample" mechanism of PF. As long as there are still several particles in any of the four sub-particle sets that can "catch" the object, all the particles can move to the object quickly, so the tracker can still keep tracking it in this situation. As a result, the four particle sets strategy was used in this paper. 

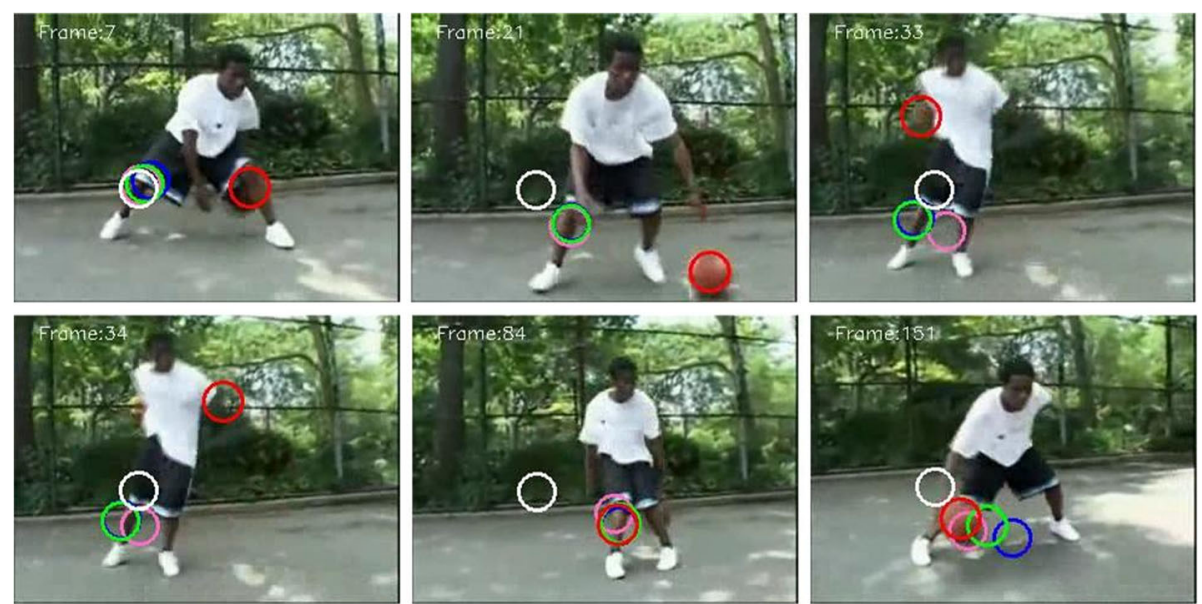

Fig. 15 The tracking results for the video "basketball_2" using CAM-Shift (white), PF (blue), VAPF (green), M-PF (pink), and the proposed tracker (red). Particle number $N=250$

In this method, there are five different particle sets in the tracker, including one main particle set and four sub-particle sets (up, down, left, and right sub sets). All four sub-particle sets are generated by the main set and distributed as the main one is, except for the particle positions. Take the rectangular template model as an example, as is described in Figs. 6 and 7.

Figure 8 shows the particle weight distribution of PF (a), VAPF (b), M-PF (c), and proposed (d) of the 7th frame of the video "tennis." It can be seen that the particles in PF are concentrated in a relatively small region and that VAPF propagates the particles in a larger scope, while the proposed tracker not only has five concentrated regions but also propagates in a larger scope (Fig. 9).

\section{Parameter A of the dynamic model}

The equation of the circular model can be specified as follows:

$$
\left[\begin{array}{l}
x_{t} \\
y_{t} \\
r_{t}
\end{array}\right]=\left[\begin{array}{lll}
A_{t}^{x} & 0 & 0 \\
0 & A_{t}^{y} & 0 \\
0 & 0 & A_{t}^{r}
\end{array}\right]\left[\begin{array}{l}
x_{t-1} \\
y_{t-1} \\
r_{t-1}
\end{array}\right]+\left[\begin{array}{lll}
B_{t}^{x} & 0 & 0 \\
0 & B_{t}^{y} & 0 \\
0 & 0 & B_{t}^{r}
\end{array}\right]\left[\begin{array}{l}
w_{t-1} \\
w_{t-1} \\
w_{t-1}
\end{array}\right]
$$

The experiment results are shown in Fig. 10a, where the parameter $A=1$ means $A_{t}^{x}=A_{t}^{y}=A_{t}^{r}=1$. It can be seen that the tracker performs best when the parameter $A_{t}^{x}=A_{t}^{y}=A_{t}^{r}=1$, because the parameter $A$ in Eq. (23) shows the relationship between the
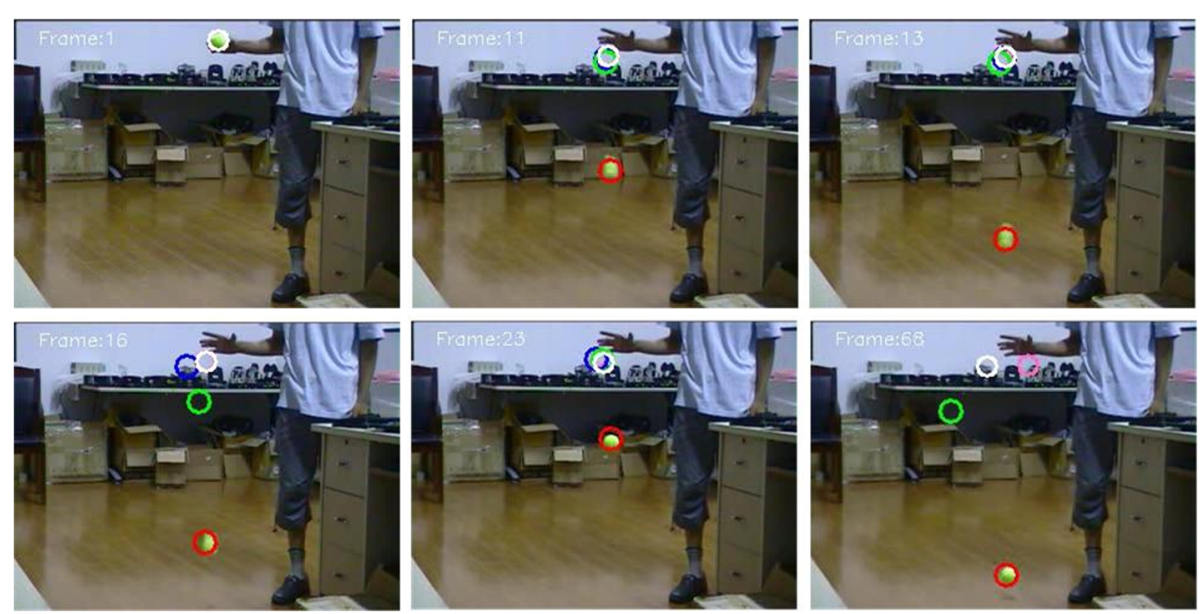

Fig. 16 The tracking results for the video "tennis" using CAM-Shift (white), PF (blue), VAPF (green), M-PF (pink), and the proposed tracker (red). Particle number $N=200$ 

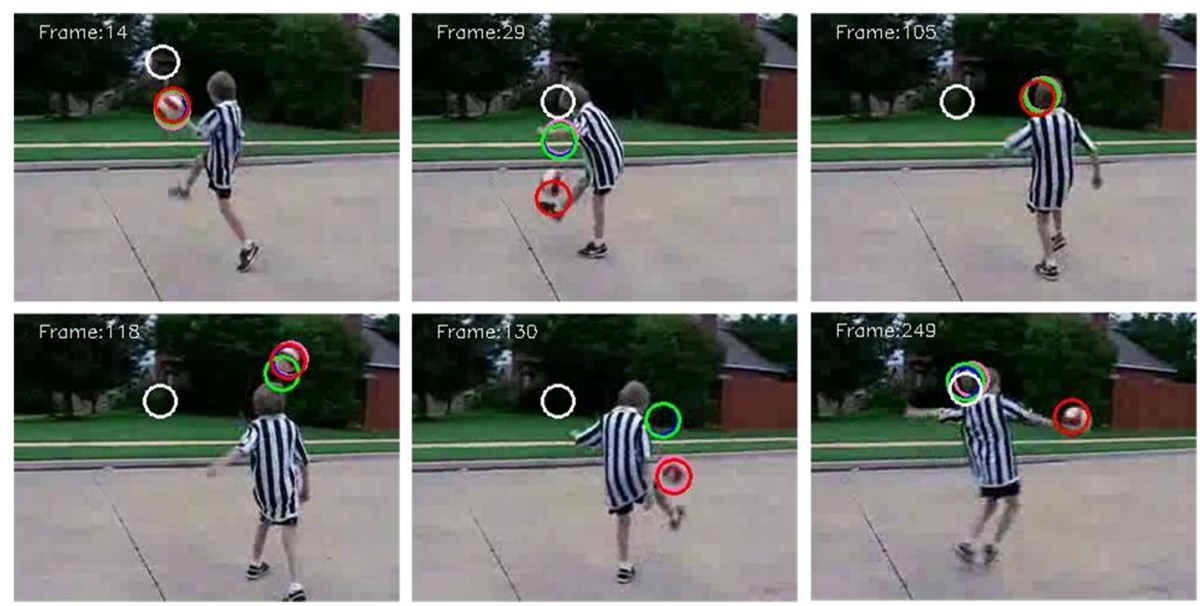

Fig. 17 The tracking results for the video "V_juggle_11_06" using CAM-Shift (white), PF (blue), VAPF (green), M-PF (pink), and the proposed tracker (red). Particle number $N=200$

object states of the current frame and the next one. Assuming that the position of one particle in the current frame is $(100,50)$, under the condition $A_{t}^{x}$ $=A_{t}^{y}=1$, the particle is propagated to $(100+$ random_x, $50+$ random_y) in the next frame, where random_x and random_y are random values decided by the estimated velocity and acceleration. In contrast, under the condition $A_{t}^{x}=A_{t}^{y}=0.5$, the particle is propagated to $(0.5 \times 100+$ random_x, $0.5 \times 50+$ random_y), and it is obvious that the propagation process is not appropriate, so the tracking tends to fail.

\section{Acceleration threshold}

From the acceleration threshold curve of Fig. 10b, it can be found that, for the video sequence "Basketball_1," the acceleration threshold between 3 and 7 pixels per frame ${ }^{2}$ tends to have a better tracking performance, while, for the video sequence "Basketball_2," all the tested thresholds are equally good. One explanation is that, in "Basketball_1," the basketball moved much more dramatically. Most of the time, the ball moved from the top to the bottom of the image within 10 frames, having the video height of 240 pixels. According to the $s_{i}=\frac{1}{2} g_{i} t_{i}^{2}$, the image acceleration $g_{i}$ was more than 2 pixels per frame ${ }^{2}$.

\section{Particle number}

For performance evaluation and comparison, all the four PF-based algorithms were tested using different numbers of particles, including 100, 150, 200, 250, and 300 , and some of the tracking results are shown in Table 1 . It can be found that the tracking performance improves when the particle number increases, and that it reaches the top when the particle number is larger than 200.
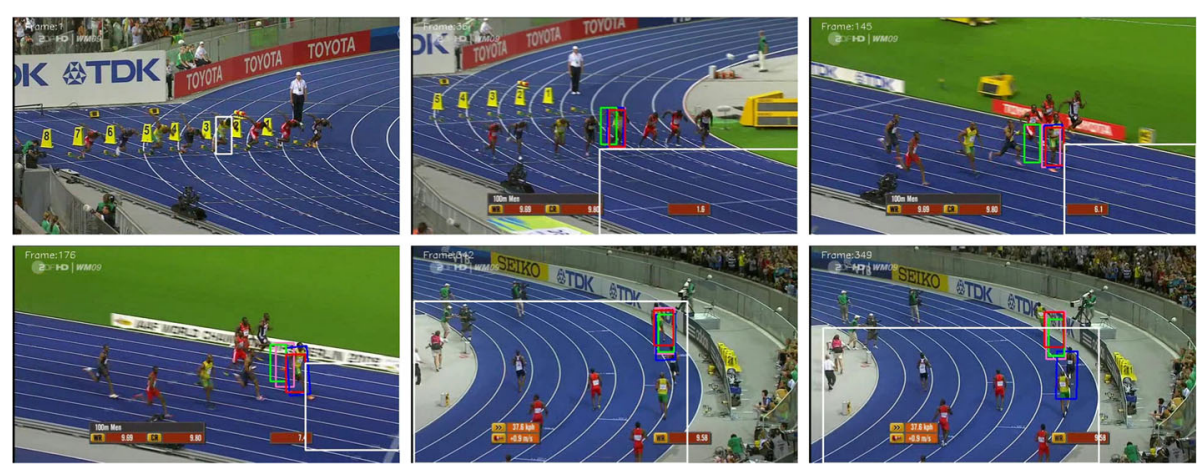

Fig. 18 The tracking results for the video "Bolt" using CAM-Shift (white), PF (blue), VAPF (green), M-PF (pink), and the proposed tracker (red). Particle number $N=200$ 

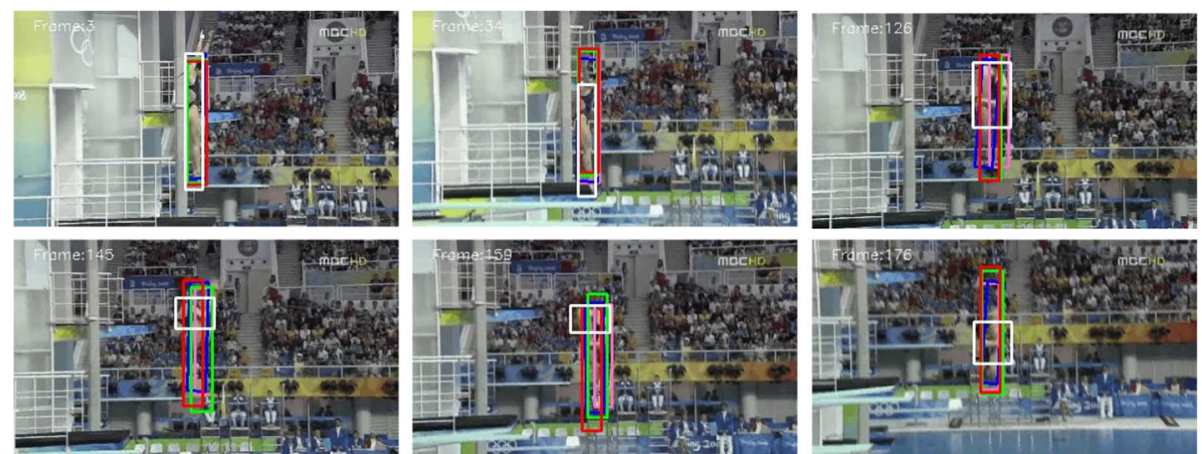

Fig. 19 The tracking results for the video "Diving" using CAM-Shift (white), PF (blue), VAPF (green), M-PF (pink), and the proposed tracker (red). Particle number $N=200$

Other parameters used in the test are shown in Table 2.

The following section first presents all the tracking results of the five algorithms in the same tested video with different color bounding boxes or circles and then performs detailed evaluations and comparisons to demonstrate the effectiveness of the algorithms.

\subsection{Performance and results overview}

To better evaluate and analyze the strength and weakness of the tracking approaches, the videos were categorized with four attributes based on the challenging factors including occlusion (OCC), background clutters (BC), affine transformation (AT), and abrupt motion (AM), as is seen in Table 3.

\subsubsection{Occlusion}

In the video sequences with the occlusion attribute, in general, all the four PF-based algorithms could recover from occlusion and loss when the object moved without abrupt motions, while differences showed up when the object moved abruptly.

In the video sequence "motinas_chamber," the tracked object was a man in red. It can be observed in Fig. 11 that, in the beginning, when the object ran slowly without occlusion, all tested algorithms tracked the object well, as can be seen in the 33rd frame, while, in the 93rd frame, when the object was occluded by another person, the proposed tracker tracks the object better not only in position but also in size due to its accurate motion estimation. When the object disappeared from the field of view in the 717th frame and showed up again in the 746th, all the four PF-based algorithms recovered from loss, but, still, the proposed tracker performed a little better.

Tracking the basketball during dribbling was a very challenging task. Moreover, the partial or total occlusion made the tracking much more difficult when the player dribbled the ball behind the back or between the legs, as can be seen in the video "basketball_2." In the 7th frame, when the basketball was blocked by his leg, the CAMShift and the PF lost its position, while the VAPF and the proposed tracker kept tracking it. And in the 33rd and 34th frames, when dribbled behind the back, the basketball was blocked by the back, but the proposed tracker had predicted this correctly and kept tracking the ball when it appeared again.
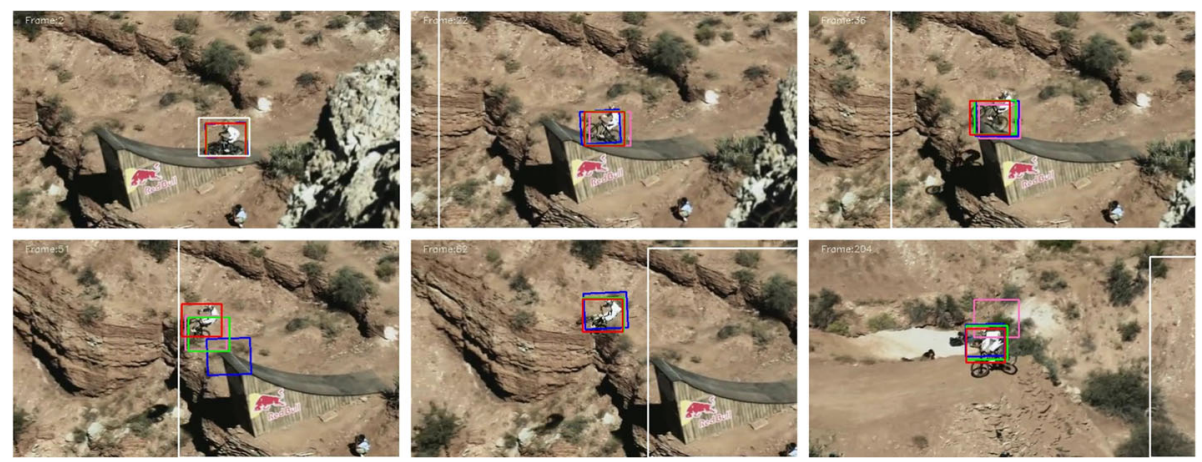

Fig. 20 The tracking results for the video "MountainBike" using CAM-Shift (white), PF (blue), VAPF (green), M-PF (pink), and the proposed tracker (red). Particle number $N=200$ 

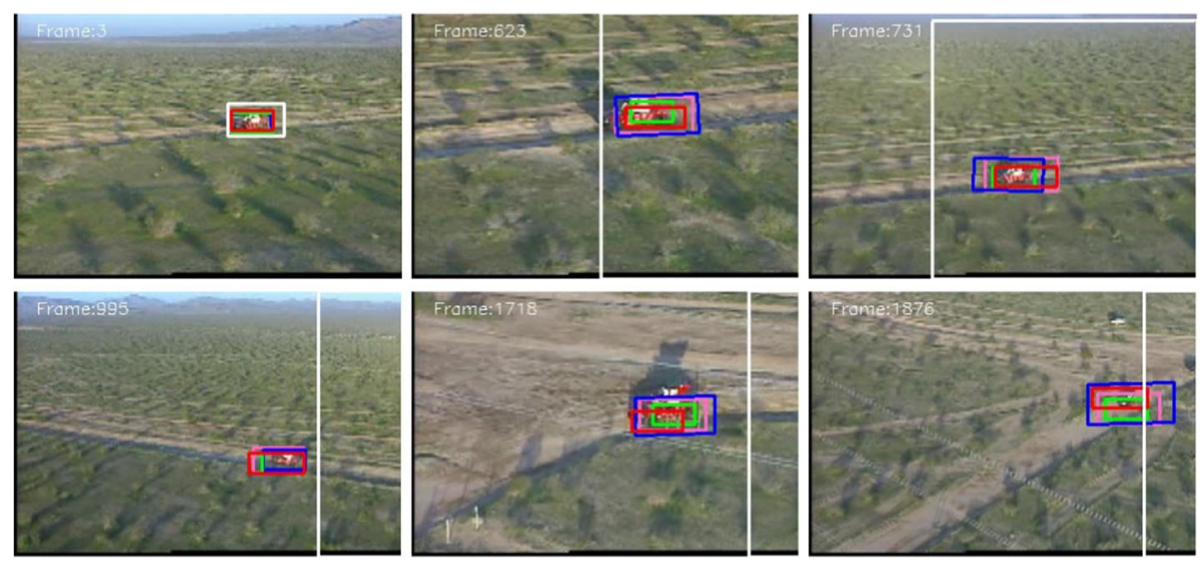

Fig. 21 The tracking results for the video "RedTeam" using CAM-Shift (white), PF (blue), VAPF (green), M-PF (pink), and the proposed tracker (red). Particle number $N=200$

\subsubsection{Background clutters}

As all the four tested algorithms were based on color distribution, they could address the background clutter problem well in most cases. However, the weakness of color distribution showed up when there was another object in almost the same color as the true target, as can be seen in the video "basketball_3," which will be discussed in the section 4.2.3.

\subsubsection{Affine transformation}

In the video sequences with the affine transformation attribute, all four PF-based trackers could deal with the factor, while CAM-Shift's bounding box was not tight due to its inability to follow the direction.

The video sequence "bottle" (Fig. 12) contained a fast moving bottle with affine transformation in a relatively simple background. Its dynamic model and background complexity was the simplest in all of the 13 tested videos. At the beginning of the video sequence, the bottle moved with a relatively low speed and small acceleration, as is shown in the 4th frame. All of the five tested algorithms performed almost equally well by tracking the object with the right position and rotation angle. When a relatively higher speed and bigger acceleration took place, as is shown in the 31st, 45th, and 46th frames, the VAPF and the proposed tracker showed their advantages compared with the CAM-Shift and the PF which could not follow the bottle well. Besides, the M-PF did not show any advantage because the video sequence was too short and did not have much history data for constructing a better prior distribution.

\subsubsection{Abrupt motion}

In the video sequences with the abrupt motion attribute, including "book," "basketball_1," "basketball_2," "basketball_3," "v_juggle_11_06," "v_juggle_15_02," and "Bolt," the proposed algorithm performed the best because the velocity and acceleration was fully taken into account in the motion-adaptive model.
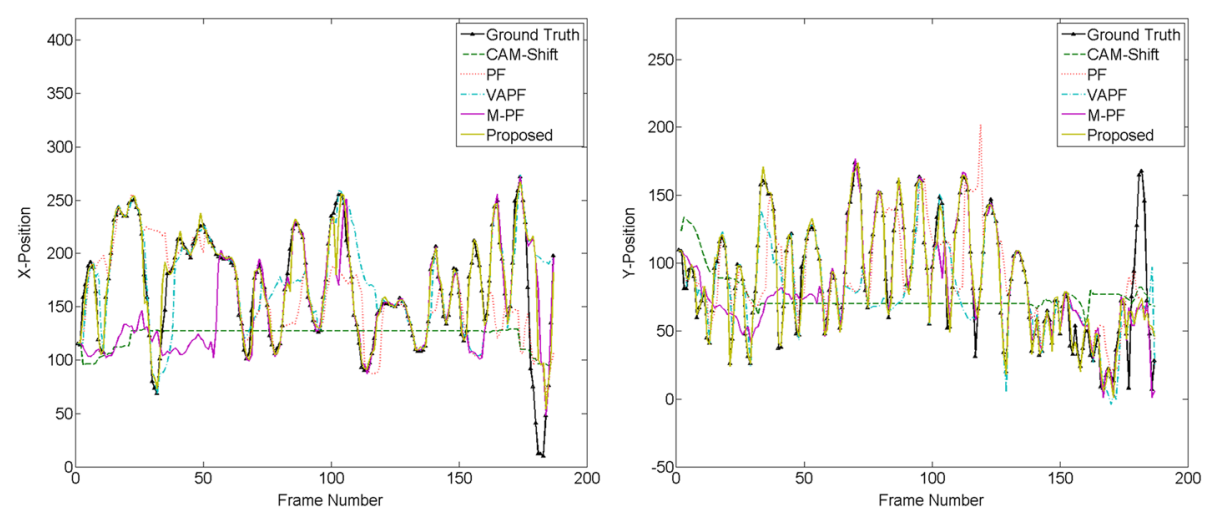

Fig. 22 Estimated object center position of "basketball_2" compared with ground truth with the particle number $N=250$ 

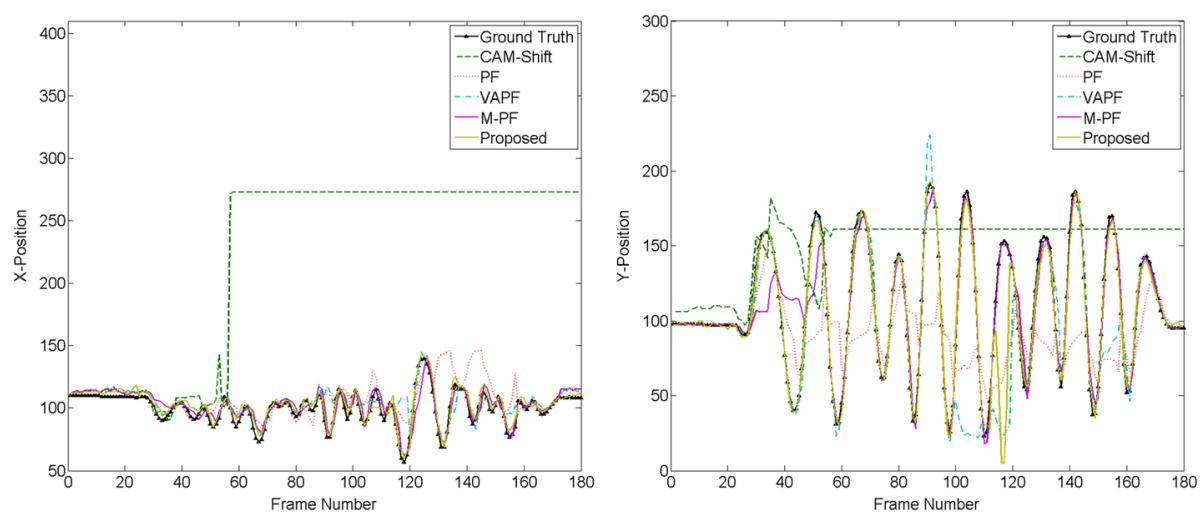

Fig. 23 Estimated object center position of "book" compared with ground truth with the particle number $N=150$

In the video sequence "book," as can be seen in Fig. 13, the CAM-Shift lost track of the object in most of the 180 frames, except for the first few frames. Although the VAPF performed better than the PF, it still lost the object in a lot of frames, especially when a sudden direction change occurred, as can be seen in the 91st frame. The M-PF did not follow the object well in the beginning because few history data were available, but performed much better after about 60 frames, as can be seen in the 91st, 124th, and 152nd frames. In comparison, the proposed tracker managed to maintain smooth tracking despite sudden orientation changes and large acceleration (Figs. 14, 15, 16, and 17).

In the video "tennis," the tennis ball first underwent a free fall and then bounced up several times. In the first few frames, all four algorithms tracked it well due to the low speed, while, in the later frames, they were affected by the acceleration of gravity, with the only exception of the proposed tracker which kept following the ball

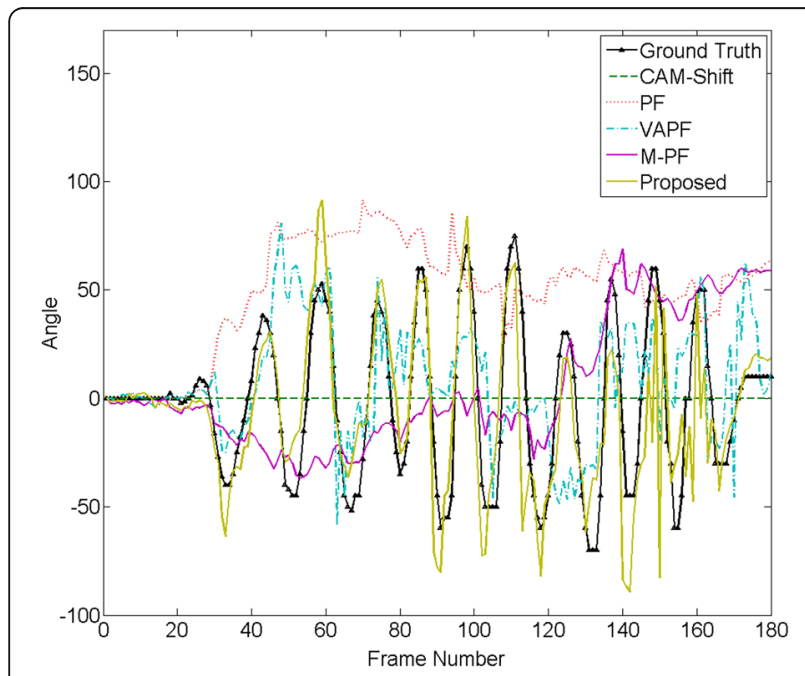

Fig. 24 Estimated object rotation angle of "book" compared with ground truth with the particle number $N=250$ because the acceleration had been fully taken into account in the motion-adaptive model. The video "v_juggle_11_06" was even more challenging than the previous three because of the similarity in color between the soccer and the background, as well as the long-time total occlusion. From the 105th frame to the 117th, the soccer was totally occluded by the kid, during which all the four trackers kept searching around the position where they lost tracking, as can be seen in the 105th frame. When the ball appeared again, the four PF-based trackers recovered from loss and went on with the tracking. In contrast, the proposed tracker performed the best in fitting the right position and size, while the CAM-Shift got totally lost (Figs. 18, 19, 20, and 21).

\subsection{Comparative performance analysis}

The following section compares the performance of the MAPF with the four analyzed trackers using Euclidian distance, that is, the distance between the tracking position and the ground truth which is manually marked, to evaluate the tracking position accuracy.

\subsubsection{Euclidian distance}

Comparisons were made between the estimated tracking position, rotation angle, width, height, and the corresponding ground truth can be seen in Figs. 22, 23, 24, and 25. The results showed that the CAM-Shift performed the worst because it lost track of the object except in the first frames, and could not recover. The PF and the VAPF did maintain tracking, but could not make an accurate estimation of position, angle, or size, while the proposed tracker followed the position of the object in both $X$ and $Y$ directions in most of the frames and fitted the object well both in rotation angle and width. However, the height result did not fit the ground truth very well because, in the video sequence of "book," the height changed dramatically due to camera view and perspective. Besides, the tracking algorithm did not use 

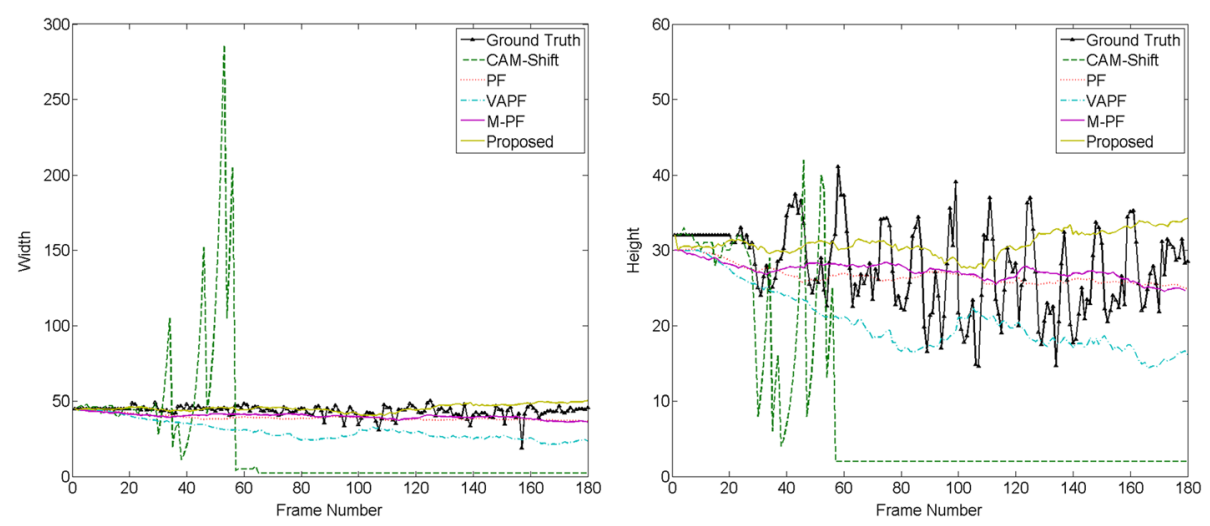

Fig. 25 Estimated width and height of "book" compared with ground truth with the particle number $N=250$

the same acceleration estimation strategy as the position related parameter in the target affine transformation.

Table 4 shows the result of the averaged Euclidian distance $\mathrm{d} 1$ over all frames in all videos from both the proposed tracker and the four analyzed trackers, while Table 5 shows the $\mathrm{d} 1$ over all frames in each video from the two groups. For the four PF-based trackers, each data is the average value of the $100,150,200,250$, and 300 particles. The table showed that the PF-based tracker always performed better than the CAM-Shift except in some special cases, such as in "Basketball_1." One explanation is that both the CAM-Shift and the PF lost the track of the object entirely, and, as a result, the positions of these two trackers tended to be random values, with one being possibly larger than the other. The performance of the proposed tracker was superior in all videos except the "bottle," because the bottle moved relatively slowly and the background was simple, as can be seen in Fig. 12 .

The rotation angle error between the tracking angle at time $t$ and the ground truth angle can be described as:

$$
e 1_{t}=\left|\theta_{t}-\theta_{t}^{G T}\right|
$$

where $\theta_{t}$ is the rotation angle in one certain algorithm at time $t$ and $\theta_{t}^{G T}$ is the ground truth in the same frame.

The error between the estimated width at time $t$ and the ground truth width can be described as:

$$
e 2_{t}=\left|W_{t}-W_{t}^{G T}\right|
$$

where $W_{t}$ is the width of the tracked object in one certain algorithm at time $t$ and $W_{t}^{G T}$ is the ground truth in the same frame.

Table 4 Resulting of averaged position error of all test videos

\begin{tabular}{llllll}
\hline Algorithms & CAM-Shift & PF & VAPF & M-PF & Proposed \\
\hline Position error & 90.99 & 34.35 & 28.33 & 25.28 & 14.75 \\
\hline
\end{tabular}

The error between the estimated height at time $t$ and the ground truth height can be described as:

$$
e 3_{t}=\left|H_{t}-H_{t}^{G T}\right|
$$

where $H_{t}$ is the height of the tracked object in one certain algorithm at time $t$ and $H_{t}^{G T}$ is the ground truth in the same frame.

Tables 6, 7, and 8 show the results of the averaged angle error e1, width error e2, and height error e3 over all frames in each video from the proposed tracker and four analyzed trackers. For the four PF-based trackers, each data is the average value of the results of 100,150 , 200, 250, and 300 particles. The videos used for the circular template model were not analyzed in these three tables because there were no "angle," "width," and "height" in the model. These three tables show that the differences between the proposed tracker and the four analyzed trackers in the "book" were much more obvious than those in the "bottle" and "motinas_chamber," because the objects in the latter two videos moved

Table 5 Resulting of averaged Euclidian distance

\begin{tabular}{llllll}
\hline Video & CAM-Shift & PF & VAPF & M-PF & Proposed \\
\hline Book & 138.83 & 40.27 & 29.06 & 10.88 & 6.52 \\
Bottle & 27.78 & 25.88 & 22.98 & 26.24 & 20.57 \\
Motinas_chamber & 67.97 & 18.87 & 16.82 & 24.48 & 13.07 \\
Bolt & 134.48 & 14.21 & 28.36 & 16.80 & 13.73 \\
Diving & 11.40 & 29.64 & 22.38 & 30.70 & 28.78 \\
MountainBike & 233.89 & 14.23 & 12.86 & 15.63 & 13.98 \\
RedTeam & 60.86 & 8.2 & 8.27 & 8.68 & 9.44 \\
Basketball_1 & 78.58 & 80.23 & 57.10 & 17.20 & 16.58 \\
Basketball_2 & 75.04 & 46.70 & 40.07 & 38.57 & 17.82 \\
Tennis & 116.31 & 78.48 & 52.67 & 64.24 & 2.17 \\
v_juggle_11_06 & 55.78 & 21.20 & 21.11 & 24.70 & 19.64 \\
\hline
\end{tabular}


Table 6 Resulting of averaged angle error

\begin{tabular}{llllll}
\hline Video & CAM-Shift & PF & VAPF & M-PF & Proposed \\
\hline Book & 28.22 & 53.56 & 28.94 & 33.7 & 16.78 \\
Bottle & 8.53 & 16.35 & 10.23 & 17.33 & 11.02 \\
\hline
\end{tabular}

relatively slowly and did not have obvious affine transformation. Nevertheless, the trends in these tables still confirmed the robustness of the proposed tracker.

\subsubsection{Time consumption}

To evaluate the computational efficiency, the time used by every algorithm was recorded. For example, in the video "basketball_1," as is seen in Fig. 26, when there were 150 particles, the PF and the VAPF needed about $60 \mathrm{~ms}$ per frame, the proposed tracker needed $50 \mathrm{~ms}$, and the CAM-Shift only needed less than $1 \mathrm{~ms}$. The statistics showed that the proposed tracker was the most efficient among the four PF-trackers, saving about 16.7\% time compared to the PF and the VAPF.

In the ordinary $\mathrm{PF}$, every state vector $\{x, y, w, h, \theta\}$ of each particle in, for example, the rectangular model is generated by a random number generator, and every cycle involves a float number operation, which is really time-consuming. Compared with that, in the proposed method of the research, only the particles of the main region are generated by the random number generator, that is, $1 / 5$ particles, while the other four regions are simply "duplicates" from the main region with the same distribution, which only need to change the position data $\{x, y\}$. The addition and subtraction operations are faster than the random number generating operation, making this method faster than the ordinary PF.

The time consumed by the M-PF tested in our experiments was much more than Mikami's proposed in [18], because the latter's algorithm could run 30.0 frames per second by using 2000 particles. The first reason for this large difference is that they employed the graphics processing unit (GPU) processing to accelerate the weight computation of particles, which was said to be 10 times faster than the CPU-only version used in the experiments of this research. Secondly, the Intel Core2Extreme 3.0GHz (Quad Core) CPU of the PC they used was much better. Therefore, the two hardware differences caused the huge difference in time consumption.

Table 7 Resulting of averaged width error

\begin{tabular}{llllll}
\hline Video & CAM-Shift & PF & VAPF & M-PF & Proposed \\
\hline Book & 36.65 & 5.09 & 13.41 & 4.27 & 3.12 \\
Bottle & 13.45 & 2.17 & 2.11 & 3.05 & 2.08 \\
Motinas_chamber & 196.21 & 6.86 & 6.54 & 11.28 & 6.50 \\
\hline
\end{tabular}

Table 8 Resulting of averaged height error

\begin{tabular}{llllll}
\hline Video & CAM-Shift & PF & VAPF & M-PF & Proposed \\
\hline Book & 19.19 & 7.39 & 5.34 & 4.41 & 4.37 \\
Bottle & 16.16 & 3.21 & 3.08 & 3.13 & 2.96 \\
Motinas_chamber & 59.73 & 17.03 & 17.05 & 29.23 & 15.44 \\
\hline
\end{tabular}

The proposed tracker, however, consumed much more time than the CAM-Shift, which was a common problem of PFs. Fortunately, the development of computer technology has provided many ways to solve the time consuming problem, such as to use parallel computing enabled by multi-core processors [25-28] and to use hash coding techniques to improve the efficiency $[29,30]$.

\subsubsection{Failure modes}

In the tests, two failure modes were identified. In the first mode, there was another object which had almost the same template and motion parameters as the tracked one, as is seen in Fig. 27, while, in the second, the tracked object had similar color distribution as part of the background, as is seen in Fig. 28.

As all the four tested algorithms were based on color distribution, when there were nearby objects in the identical color with the selected one or the background color distribution was similar to the object's, the tracking process would probably end up with failure. In the first example, the man was dribbling two basketballs at the same time, and occlusion often took place when dribbling behind the back or between legs, as can be seen in the 23rd and 83rd frames. In the 23rd frame, when the tracked basketball disappeared, the proposed tracker moved to another basketball immediately while the other

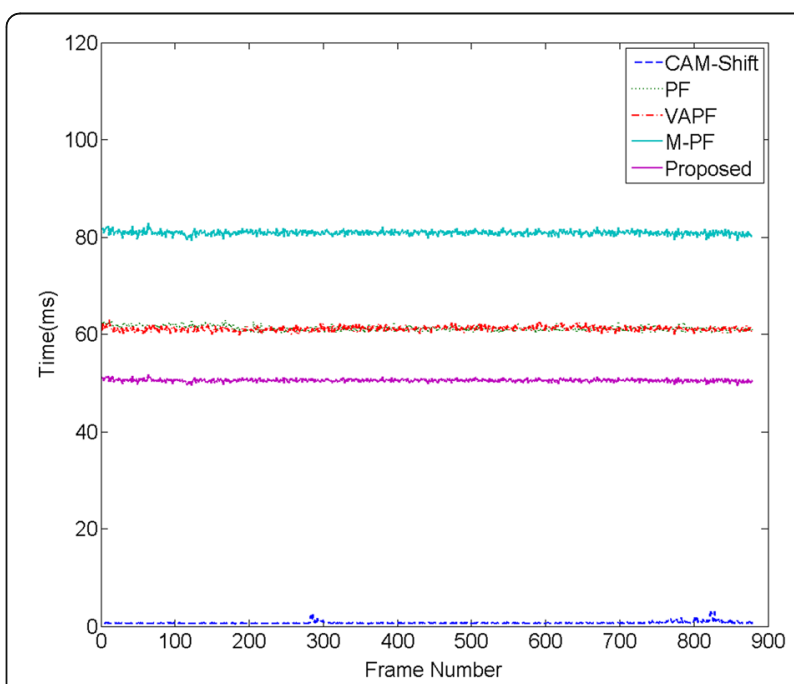

Fig. 26 Resulting of time consuming of video "basketball_1" with the particle number $N=150$ 

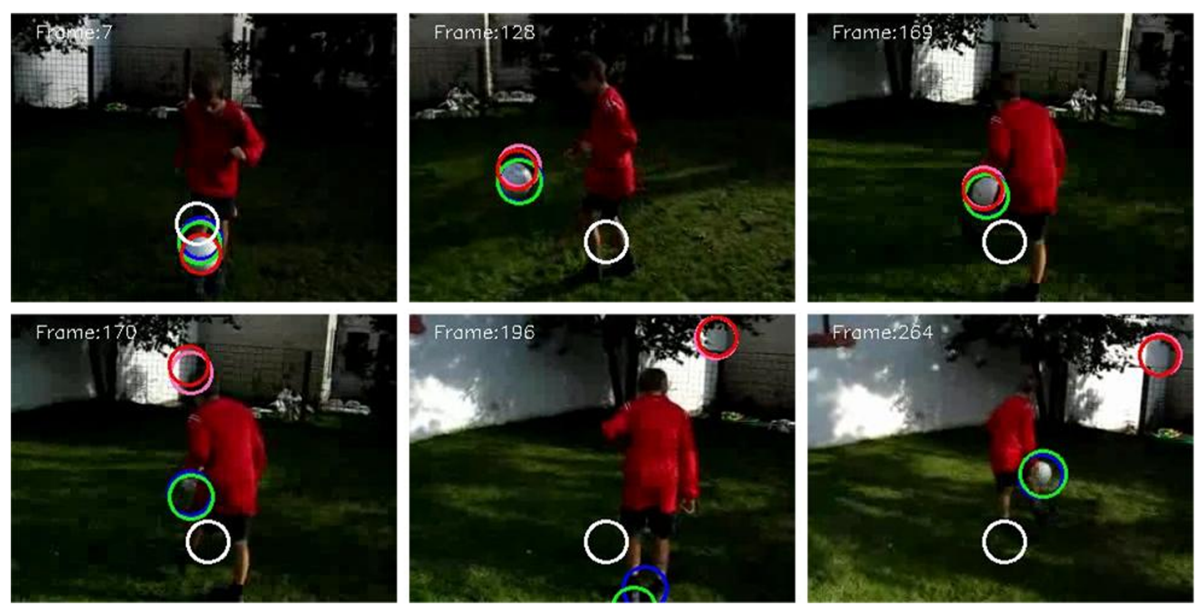

Fig. 27 The tracking failure example of video "v_juggle_15_02" using CAM-Shift (white), PF (blue), VAPF (green), M-PF (pink), and the proposed tracker (red). Particle number $N=250$

four tended to stay in the original position and search, because the SPD mechanism of the proposed tracker detected a dramatic movement due to the similarity between the two basketballs. This was also the reason for the failure in the 170th, 196th, and 224th frames of the video "v_juggle_15_02," which could be avoided by combing a much better appearance mode and object correspondence mechanism. However, finding a good template model for visual tracking remains a challenging task.

\section{Conclusion}

This paper has presented a MAPF for visual object tracking under complex dynamics. The ways of using the prior history position data to estimate the velocity and acceleration of moving objects were demonstrated, and a new method called SPD was proposed to improve the robustness of tracking a fast-moving object. Through updating the propagation distance in the dynamic model in each frame, the robustness and effectiveness were significantly improved during the tracking process. The experimental results demonstrated that, compared with the CAM-Shift, PF, VAPF, and M-PF, the proposed algorithm was effective and robust in dealing with object tracking under conditions of complex dynamics, occlusion, and affine transformation. The tracking performance was improved significantly not only in position accuracy and object similarity, but also in computational efficiency.

The proposed algorithm was inspired by the colorbased PF and has turned out to be better, and it will be helpful in other PF algorithms that need to consider
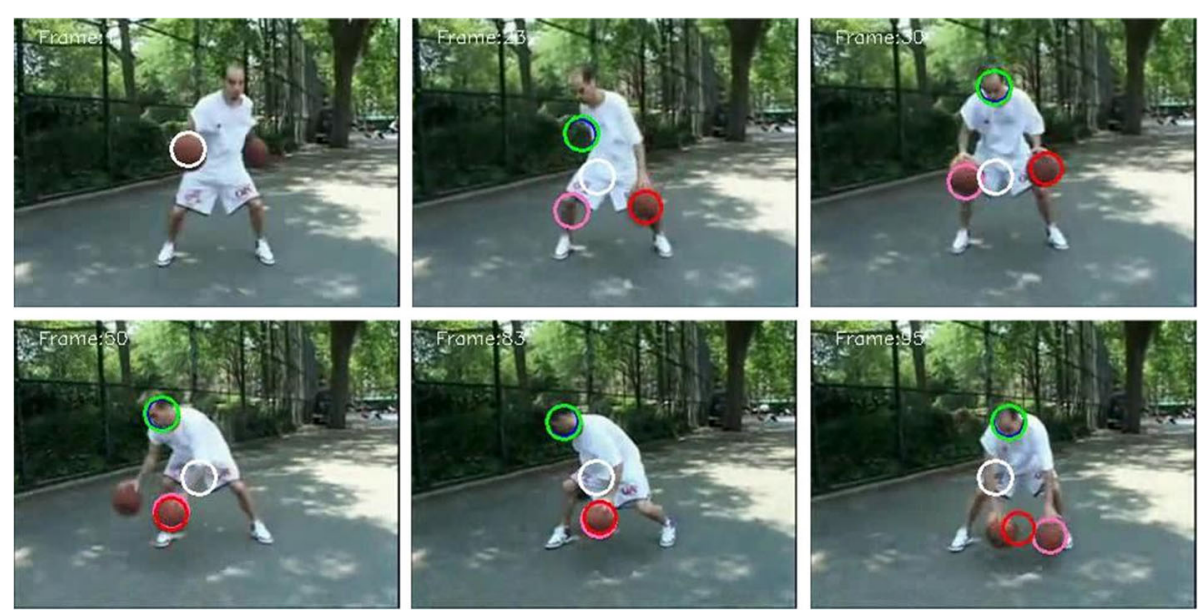

Fig. 28 The tracking failure example of video "basketball_3" using CAM-Shift (white), PF (blue), VAPF (green), M-PF (pink), and the proposed tracker (red). Particle number $N=200$ 
objects moving with dramatic changes in velocity and acceleration.

\section{Additional files}

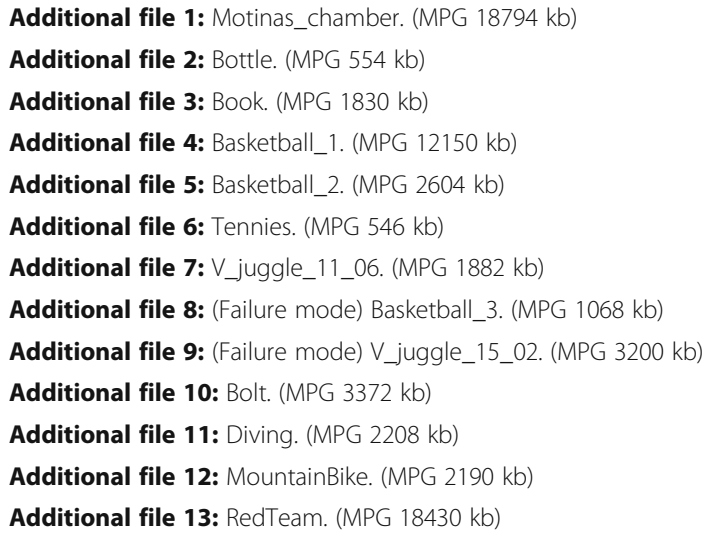

\section{Abbreviations}

CAM-Shift: Continuously Adaptive Mean-Shift; MAPF: Motion-Adaptive Particle Filter; M-PF: Memory-based Particle Filter; PF: Standard particle filter; SPD: Sub-particle drift; VAPF: Velocity-Adaptive Particle Filter

\section{Acknowledgements}

The most heartfelt gratitude goes to Changwei Wu and Houqiang Zhao for their helpful discussion and feedback about the algorithm, as well as Fangge Lu for proofreading the English writing.

\section{Funding}

This research was supported in part by the Natural Science Foundation of China (NSFC) (Grant No: 51175459).

\section{Availability of data and materials}

The web links to the sources of the data (namely, images) used for our experiments and comparisons in this work have been provided in this article.

\section{Authors' contributions}

SC implemented the core algorithm, designed all the experiments, addressed the resulting data, and drafted the manuscript. XW participated in the design and construction of the motion-adaptive model and helped draft the manuscript. KX implemented the color distribution model and object template model and participated in designing the PF core algorithm. All authors have read and approved the final manuscript.

\section{Ethics approval and consent to participate}

Not applicable.

\section{Consent for publication}

Not applicable.

\section{Competing interests}

The authors hereby declare that they have no competing interests.

\section{Publisher's Note}

Springer Nature remains neutral with regard to jurisdictional claims in published maps and institutional affiliations.
Received: 24 September 2016 Accepted: 2 November 2017

Published online: 21 November 2017

\section{References}

1. S Khan, M Shah, A Multiview Approach to Tracking People in Crowded Scenes Using a Planar Homography Constraint, Computer Vision-ECCV 2006 (Springer, Berlin,Heidelberg, 2006), pp. 133-146

2. A Yilmaz, Object tracking: a survey. ACM Comput. Surv. 38(4), 81-93 (2006)

3. K. Cannons, A review of visual tracking. Dept. Comput. Sci. Eng. (2008)

4. NJ Gordon, DJ Salmond, AFM Smith, Novel approaches to nonlinear/nonGaussian Bayesian state estimation. IEE Proc. F-Radar Signal Process 140(2), 107-113 (1993)

5. S Maskell, N Gordon, A tutorial on particle filters for on-line nonlinear/nonGaussian Bayesian tracking. Target Track. Algorithms Appl 2, 2/1-2/15 (2001)

6. K Nummiaro, E Koller-Meier, LV Gool, An adaptive color-based particle filter. Image Vis. Comput 21(1), 99-110 (2003)

7. Y Li, HZ Ai, T Yamashita, SH Lao, M Kawade, Tracking in low frame rate video: a cascade particle filter with discriminative observers of different life spans. IEEE Trans. Pattern Anal. Mach. Intell. 30(10), 1728-1740 (2007)

8. J Kwon, KM Lee, FC Park, Visual tracking via geometric particle filtering on the affine group with optimal importance functions. IEEE Conf. Comput. Vis. Pattern Recognit 29, 991-998 (2009)

9. ZH Khan, IYH Gu, AG Backhouse, A robust particle filter-based method for tracking single visual object through complex scenes using dynamical object shape and appearance similarity. J. Signal Proc. Syst. Signal Image Video Technol 65(1), 63-79 (2011)

10. M Isard, A Blake, Contour tracking by stochastic propagation of conditional density. Eur. Conf. Comput. Vis 1064, 343-356 (1996)

11. B North, A Blake, M Isard, J Rittscher, Learning and classification of complex dynamics. IEEE Trans. Pattern Anal. Mach. Intell. 22(9), 1016-1034 (2000)

12. R Hess, A Fern, Discriminatively trained particle filters for complex multiobject tracking. IEEE Conf. Comput. Vis. Pattern Recognit, 240-247 (2009)

13. Y Wu, TS Huang, A co-inference approach to robust visual tracking. Eighth IEEE Int. Conf. Comput. Vis 2, 26-33 (2001)

14. MD Breitenstein, F Reichlin, B Leibe, E Koller-Meier, L Van Gool, Robust tracking-by-detection using a detector confidence particle filter. IEEE 12th Int. Conf. Comput. Vis, 1515-1522 (2009)

15. SHK Zhou, R Chellappa, B Moghaddam, Visual tracking and recognition using appearance-adaptive models in particle filters. IEEE Trans. Image Process. 13(11), 1491-1506 (2004)

16. YM Lui, JR Beveridge, LD Whitley, Adaptive appearance model and condensation algorithm for robust face tracking. IEEE Trans. Syst. Man Cybern. Part a-Syst. Hum 40(3), 437-448 (2010)

17. A Del Bimbo, F Dini, Particle filter-based visual tracking with a first order dynamic model and uncertainty adaptation. Comput. Vis. Image Underst. 115(6), 771-786 (2011)

18. D Mikami, K Otsuka, J Yamato, Memory-based particle filter for face pose tracking robust under complex dynamics. IEEE Conf. Comput. Vis. Pattern Recognit, 999-1006 (2009)

19. S Yang, Particle filtering based estimation of consistent motion and disparity with reduced search points. IEEE Trans. Circuits Syst. Video Technol 22(1), 91-104 (2012)

20. B Han, Y Zhu, D Comaniciu, LS Davis, Visual tracking by continuous density propagation in sequential Bayesian filtering framework. IEEE Trans. Pattern Anal. Mach. Intell. 31(5), 919-930 (2009)

21. G. R. Bradski, Computer Vision Face Tracking For Use in a Perceptual User Interface. Intel Technology Journal, 2nd Quarter, 214-219 (1998)

22. Y Wu, J Lim, M-H Yang, Online object tracking: a benchmark. Proc. IEEE Conf. Comput. Vis. Pattern Recognit, 2411-2418 (2013)

23. UCF YouTube Action Dataset. http://vision.eecs.ucf.edu/projects/liujg/ YouTube Action dataset.html. A ccessed 11 Apr 2015.

24. SPEVI dataset. http://www.eecs.qmul.ac.uk/ andrea/spevi.html. Accessed 11 Apr 2015.

25. C. Yan, Y Zhang, J Xu, et al. A highly parallel framework for HEVC coding unit partitioning tree decision on many-core processors[]]. IEEE Signal Process. Lett, 21(5),573-576 (2014).

26. C Yan, Y Zhang, J Xu, et al., Efficient parallel framework for HEVC motion estimation on many-core processors[J]. IEEE Trans. Circuits Syst. Video Technol 24(12), 2077-2089 (2014)

27. C Yan, Y Zhang, F Dai, et al., Parallel deblocking filter for HEVC on manycore processor[J]. Electron. Lett. 50(5), 367-368 (2014) 
28. C Yan, Y Zhang, F Dai, et al., Efficient parallel HEVC intra-prediction on many-core processor[J]. Electron. Lett. 50(11), 805-806 (2014)

29. C Yan, H Xie, D Yang, J Yin, Y Zhang, Q Dai, Supervised hash coding with deep neural network for environment perception of intelligent vehicles. IEEE Trans. Intell. Transp. Syst. 99, 1-12 (2017)

30. C Yan, H Xie, S Liu, J Yin, Y Zhang, Q Dai, Effective Uyghur language text detection in complex background images for traffic prompt identification. IEEE Trans. Intell. Transp. Syst. 99, 1-10 (2017)

Submit your manuscript to a SpringerOpen ${ }^{\circ}$ journal and benefit from:

- Convenient online submission

- Rigorous peer review

- Open access: articles freely available online

- High visibility within the field

- Retaining the copyright to your article

Submit your next manuscript at $\gg$ springeropen.com 